\title{
Second-mode attenuation and cancellation by porous coatings in a high-speed boundary layer
}

\author{
Guillaume A. Brès ${ }^{1,} \dagger$, Matthew Inkman ${ }^{1}$, Tim Colonius ${ }^{1}$ \\ and Alexander V. Fedorov ${ }^{2}$ \\ ${ }^{1}$ Department of Mechanical Engineering, California Institute of Technology, 1200 E. California Blvd., \\ Pasadena, CA 91125, USA \\ ${ }^{2}$ Department of Aeromechanics and Flight Engineering, Moscow Institute of Physics and Technology, \\ Zhukovsky, 140180, Russia
}

(Received 4 December 2012; revised 2 April 2013; accepted 17 April 2013; first published online 31 May 2013)

Numerical simulations of the linear and nonlinear two-dimensional Navier-Stokes equations, and linear stability theory are used to parametrically investigate hypersonic boundary layers over ultrasonic absorptive coatings. The porous coatings consist of a uniform array of rectangular pores (slots) with a range of porosities and pore aspect ratios. For the numerical simulations, temporally (rather than spatially) evolving boundary layers are considered and we provide evidence that this approximation is appropriate for slowly growing second-mode instabilities. We consider coatings operating in the typical regime where the pores are relatively deep and acoustic waves and second-mode instabilities are attenuated by viscous effects inside the pores, as well as regimes with phase cancellation or reinforcement associated with reflection of acoustic waves from the bottom of the pores. These conditions are defined as attenuative and cancellation/reinforcement regimes, respectively. The focus of the present study is on the cases which have not been systematically studied in the past, namely the reinforcement regime (which represents a worst-case scenario, i.e. minimal second-mode damping) and the cancellation regime (which corresponds to the configuration with the most potential improvement). For all but one of the cases considered, the linear simulations show good agreement with the results of linear instability theory that employs an approximate porous-wall boundary condition, and confirm that the porous coating stabilizing performance is directly related to their acoustic scattering performance. A particular case with relatively shallow pores and very high porosity showed the existence of a shorter-wavelength instability that was not initially predicted by theory. Our analysis shows that this new mode is associated with acoustic resonances in the pores and can be more unstable than the second mode. Modifications to the theoretical model are suggested to account for the new mode and to provide estimates of the porous coating parameters that avoid this detrimental instability. Finally, nonlinear simulations confirm the conclusions of the linear analysis; in particular, we did not observe any tripping of the boundary layer by small-scale disturbances associated with individual pores.

Key words: boundary layer stability, high-speed flow, instability control

$\dagger$ Present address: Cascade Technology Inc., Palo Alto, CA 94303, USA. Email address for correspondence: guillaumebres@gmail.com 


\section{Introduction}

Delay of laminar-turbulent transition on hypersonic vehicles would enable a significant reduction of the weight and complexity of its thermal protection system by reducing the heat transfer rate to the surface. State-of-the-art active and reactive laminar flow control techniques seem to be impractical under the severe conditions of hypersonic flight (e.g. Kimmel 2003), but passive techniques that can be integrated with a thermal protection system, such as porous coatings, have been shown to significantly increase laminar run in wind-tunnel experiments (Rasheed et al. 2002). Fedorov (2011) provides a review of stability and laminar-turbulent transition in highspeed boundary-layer flows. At high Mach numbers and low wall temperature ratios on the vehicle surface, the second mode, or Mack (1969) mode, of instability in the boundary layer suffers a higher growth rate than the Tollmien-Schlichting wave (first mode). Second-mode instability has been observed and measured in many wind-tunnel experiments (e.g. Kendall 1975; Stetson et al. 1983; Stetson \& Kimmel 1992). The mode involves high-frequency (ultrasonic) acoustic waves trapped in the boundary layer (Malmuth et al. 1998). So-called ultrasonic absorptive coatings (UAC), are micro-structured surfaces designed with porosity, pore spacing, and pore depth tuned to absorb these instability waves and thus suppress second-mode growth.

Several experimental and theoretical studies (e.g. Fedorov et al. 2001, 2003a,b; Bountin et al. 2004; Maslov et al. 2006) have confirmed the UAC stabilization concept. More recently, direct numerical simulations (DNS) of both two-dimensional surfaces with regular spanwise slots (Brès, Colonius \& Fedorov 2008b; Wartemann, Lüdeke \& Sandham 2009; Hader \& Fasel 2011), and three-dimensional surfaces with streamwise slots, and rectangular pores with various shapes (Sandham \& Lüdeke 2009; De Tullio \& Sandham 2010) have investigated aspects of linear and nonlinear instability and transition of UAC.

Structural and fabrication constraints aside, the absorptivity of the UAC, over a range of frequencies, is typically maximized by having high-porosity surfaces with narrow, deep, regular pores. Following our previous studies (see Brès, Colonius \& Fedorov 2008a, 2010), the starting hypothesis of the present work is that the efficacy of the surface in absorbing second-mode instability waves may be largely inferred from the normal-incidence reflection coefficient of plane acoustic waves with the UAC. Figure 1(c) shows a nominally two-dimensional UAC surface consisting of regular, spanwise slots. The relevant parameters characterizing the reflection coefficient $\mathscr{R}$ are the acoustic frequency $f H / a_{w}$, and the porosity $\phi$, cavity aspect ratio $A R$, and acoustic Reynolds number $R e$ defined as

$$
\phi=2 b / s, \quad A R=2 b / H, \quad R e=\rho_{w} a_{w} b / \mu_{w},
$$

where the values of density $\rho$, sound speed $a$, and viscosity $\mu$ are taken at the wall, as denoted by the subscript $w$. In figure 2, the normal-incidence acoustic reflectivity is plotted for various values of $\phi$ and $A R$, over a range of frequencies relevant to UAC design. The minimum reflectance on this plot yields a maximally absorptive surface and is associated either with the attenuative effect of viscosity on the acoustic wave within the pore, which yields the decaying reflectance with increasing frequency, or, in the case of the peaks and troughs in reflectivity, with phase cancellation or reinforcement associated with reflection from the bottom of the cavities (see Brès et al. 2009). In what follows, we refer to these as the attenuative and cancellation/reinforcement regimes, respectively. Designs which have been tested to date have been in the attenuative regime associated with deep pores. However, if the UAC surface could be accurately tuned to the local frequency of the second- 
(a)

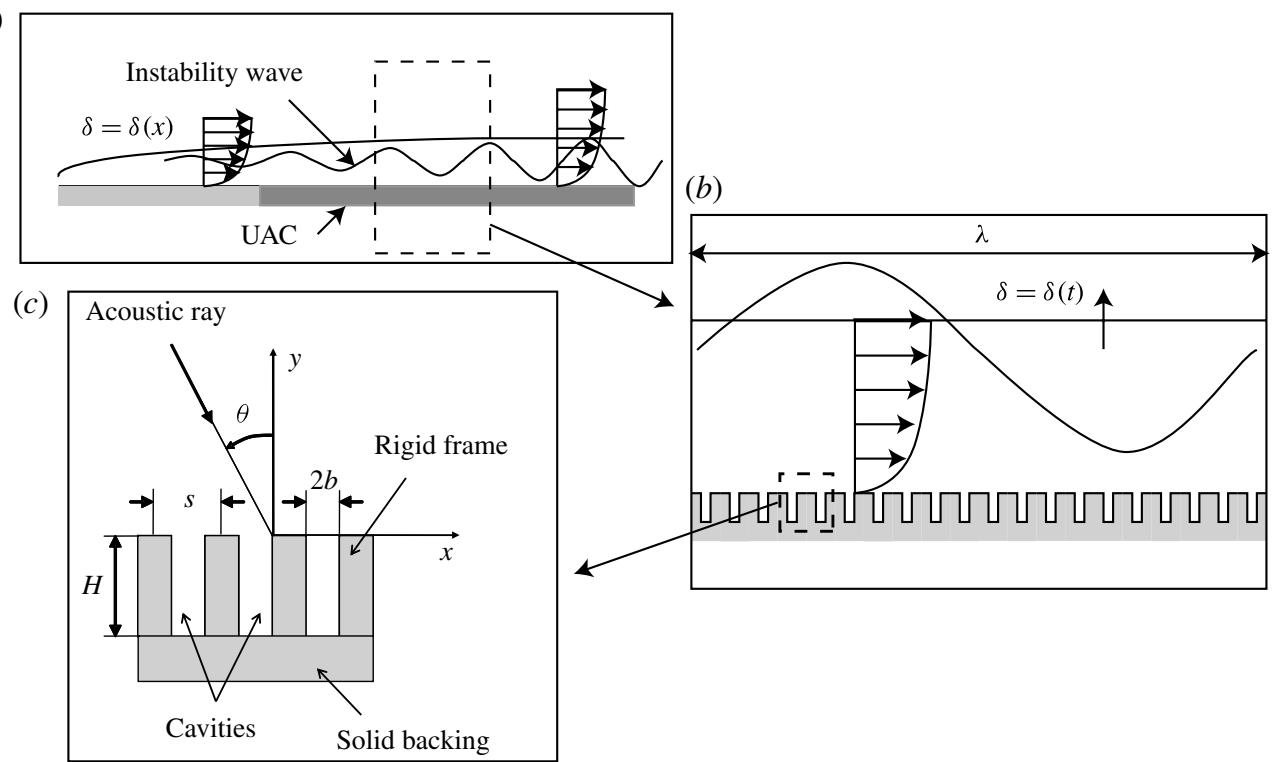

FIGURE 1. Schematic diagram for DNS of UAC (not to scale): (a) the spatial problem with many wavelengths of the spatially growing instability wave; $(b)$ the temporal problem with a single wavelength of the temporally growing instability wave; $(c)$ schematic of the reflection of an acoustic wave from equally spaced two-dimensional cavities.
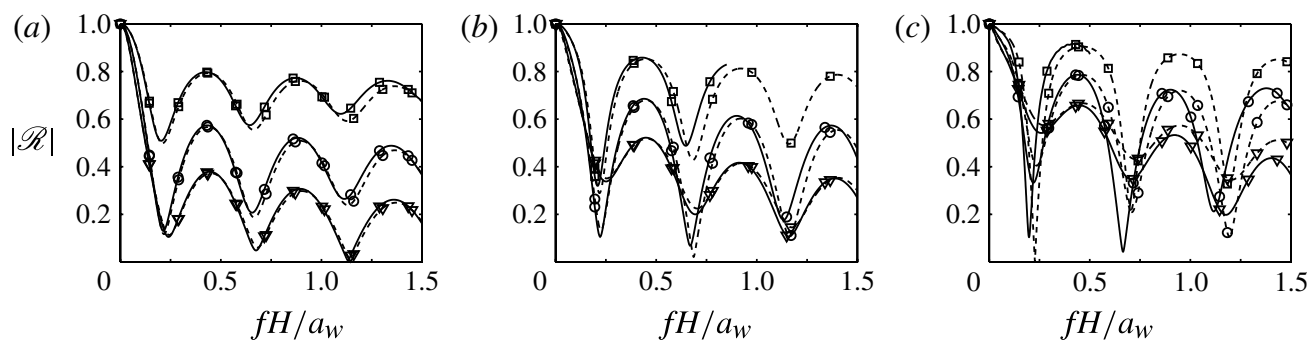

FIGURE 2. Comparison of the reflection coefficient amplitude at normal incidence from the present DNS (solid lines) and the theoretical model (dashed lines) by Kozlov, Fedorov \& Malmuth (2005) at acoustic Reynolds number $R e=100$, for coatings of porosity $\phi=0.2$ $(\square), \phi=0.48(\bigcirc)$, and $\phi=0.8(\nabla)$. (a) Cavity aspect ratio $A R=0.06$; (b) $A R=0.12$; (c) $A R=0.3$.

mode instability wave (for example by having slowly varying depth with streamwise distance), then good absorptivity could be achieved in the cancellation regime with much shallower pores over a range of porosities.

In this study, we use DNS and linear stability theory (LST) to investigate the linear (and, to a more limited extent, nonlinear) stability of the second mode over a wide range of parameters associated with both the attenuative and cancellation regimes. Our objective is twofold: first, to confirm (and potentially improve) the robustness and accuracy of existing models for the evaluation of UAC designs. Second, to confirm the absorptivity that would be implied by figure 2, especially in the cancellation regime which has not been systematically studied in past experiments or simulations. 
In order to perform DNS of the UAC, some tradeoffs are required to deduce a set of computationally tractable model problems, since a realistic surface would have as many as 20 pores per wavelength of the most unstable wave. In our previous work, a simplified configuration that considers a temporally evolving boundary layer on an infinite porous plate is shown to retain enough of the relevant flow physics in order to meet the overall modelling objectives (Brès et al. 2008a). A schematic of this configuration is shown in figure 1. Moreover, at least for the linear stability calculations, we show below that the growth rate of the second mode is sufficiently small that the Gaster (1962) transformation can be used to accurately obtain spatial growth rates and phase speeds from the temporal analysis. The temporally evolving boundary layer neglects its spatial growth, and instead diffuses slowly with time. Over short time scales associated with acoustic energy attenuation in UAC, the laminar boundary layer is essentially frozen, consistent with either a spatial or temporal description of the mean flow field, and consistent with parallel flow approximations that are typically made in instability calculations. The same temporal approximation has been invoked in latter DNS studies by other authors (Sandham \& Lüdeke 2009; Wartemann et al. 2009; De Tullio \& Sandham 2010; Hader \& Fasel 2011).

A limitation of our study is that, to enable calculations over a broad range of parameters, we restrict our attention to two-dimensional, spanwise-slot geometries and two-dimensional flows (zero spanwise wavenumber). In terms of the absorption of the second mode, this assumption is quite reasonable because the most unstable second mode is two-dimensional, and a detailed comparison of two- and three-dimensional pore geometries by Sandham \& Lüdeke (2009) led to similar growth rates for two- and three-dimensional surfaces for the same hydraulic diameter of the pores. De Tullio \& Sandham (2010) investigated the nonlinear stage of laminar-turbulent transition in a Mach 6 adiabatic flat-plate boundary layer over a porous surface comprising equally spaced cavities of rectangular cross-section. The computational domain included four pores in the streamwise and spanwise directions, giving a total of 16 pores. The porous surface was confirmed to attenuate both primary second-mode instability and secondary instabilities (through reduced amplitude of second-mode saturation), but an oblique first-mode wave is the most amplified mode in this porous surface configuration, consistent with the linear theory and experiment (Fedorov et al. $2003 a, b)$ at the adiabatic wall temperature. This wave is slightly destabilized by the pores. With the oblique first mode excited, the flow becomes turbulent due to the nonlinear interactions without the need for secondary instabilities. The linear theory suggests, however, that at the lower wall temperatures used in this study, the oblique first-mode waves are stable.

The remainder of the paper is organized as follows. The DNS and LST methodology, as well as the theoretical modelling of the acoustic properties of the coating, are discussed in $\S \S 2$ and 3 , respectively. In $\S 4$, the properties of the second-mode instability for a hypersonic boundary layer are considered for a range of UAC porosities and aspect ratios using linearized DNS, LST, and nonlinear DNS. The analysis confirms that the cancellation regime is viable for damping secondmode instability, but, at sufficiently high porosities, reveals the presence of a resonant acoustic mode of the UAC that is more unstable than the second mode, with important implications for the practical design of UAC. The physical mechanism giving rise to this mode is discussed in $\S 5$ and the theoretical model is modified to account for cavity mouth effects in order to bring the predicted growth rate of the resonant mode into agreement with the computed results. A summary of the main conclusions is presented in $\S 6$. 


\section{Methodology}

\subsection{Direct numerical simulations}

In the present work, direct numerical simulations are performed for both the nonlinear (DNS) and linearized (LDNS) two-dimensional Navier-Stokes equations. The fully compressible momentum, continuity and energy equations are solved on a blockstructured mesh, using a sixth-order compact finite-difference scheme for spatial discretization in the $x$ - and $y$-direction (Lele 1992) and a fourth-order Runge-Kutta algorithm for time-marching. Details on the code algorithm, implementation and validations are reported by Brès \& Colonius (2008).

For the temporal stability problem, periodic boundary conditions are applied in the streamwise direction, and the nominally laminar boundary layer spreads in time rather than streamwise position. The computational domain extends up to $5 H$ in the $y$-direction above the wall. The boundary conditions are non-reflective at the top boundary (Thompson 1990; Poinsot \& Lele 1992), with a buffer zone implemented to avoid spurious reflections (Colonius, Lele \& Moin 1993; Freund 1997). In preliminary studies, larger heights of the simulation domain were tested, up to $18 \mathrm{H}$, as used in our previous work on the acoustic properties of porous coatings (Brès et al. 2010). The current dimensions were chosen to reduce computational cost and the position and treatment of the outer boundary were verified not to have detrimental effects on the results. All the numerical simulations are performed on similar stretched Cartesian grids, with clustering of points near the walls. For the configuration with the porous wall, all the cavities are explicitly included in the computational domain, and the mesh contains about half a million grid points, with 100 points per cavity depth, and 12-60 points across each cavity length, depending on the pore aspect ratio.

The equations are solved for an ideal gas, with constant specific heat capacity, $\gamma=1.4$ and constant Prandtl number $\operatorname{Pr}=0.7$. The Mach number is $M_{\infty}=U_{\infty} / a_{\infty}=$ 6 , and the wall temperature $T_{w} / T_{\infty}=1.4$ is assumed to be uniform and constant. The subscript $\infty$ denotes the free-stream value of the property. Here, the choice of a relatively low wall temperature was made in order to reinforce the secondmode instability and demonstrate that this instability is effectively damped by the porous coating. Previous theoretical and experimental studies (e.g. Fedorov et al. 2001) have shown that the porous-wall stabilization technique remains effective for higher wall temperature, up to adiabatic wall temperature. Furthermore, according to an assumption of radiative equilibrium, the chosen value of $T_{w} / T_{\infty}=1.4$ would approximately correspond to $T_{\infty}=800 \mathrm{~K}$ for a laminar boundary layer of thickness $5 \mathrm{~mm}$, which are reasonable values for practical applications.

The dynamic viscosity $\mu$ and thermal conductivity $k$ are modelled as following a power-law temperature-dependence, $\mu / \mu_{\infty}=k / k_{\infty}=\left(T / T_{\infty}\right)^{n}$, with $n=1$. For predictions of UAC performance in practical cases, more realistic temperature dependence of viscosity should be used. Nevertheless, the temperature-viscosity law does not affect the basic mechanism leading to the second-mode stabilization on the porous coating. The previous theoretical studies, which were performed with the power law (Fedorov et al. 2001) with $n=0.75$, and with the Sutherland law (Fedorov et al. 2003a,b; Brès et al. 2008b; Fedorov 2010) showed the same qualitative effect. Therefore, the basic trends and conclusions in the present paper are expected to hold for other dependences. The computations are initialized with an error-function profile for the streamwise velocity (i.e. the correct self-similar solution as $M_{\infty} \rightarrow 0$ ), uniform pressure and using the Crocco-Busemann relation to compute the initial temperature profile for the chosen wall temperature ratio (Schlichting \& Gersten 2000). 
The nonlinear simulations are advanced in time until the boundary-layer thickness $\delta / H$ reaches the desired value.

The resulting boundary layer profile is then frozen and used as the base flow in linear simulations. An initial perturbation in the form of a small, Gaussian pressure pulse is added to the flow and the long-time response is recorded. To quantify nonlinear effects, the same acoustic perturbation is also added to the nonlinear simulations discussed in $\S 4.4$, but in these cases, the boundary layer continues to grow in time during the simulation.

The streamwise extent of the domain $L_{x}$ is chosen to match the predicted wavelength of the most unstable second mode as closely as possible while containing an integer number of pores, $N_{\text {pore }}$. While the typical number of pores per wavelength of the instability is suggested to be $\sim 10-20$ (see Fedorov et al. 2001), its value varies from 3 to 66 in the present simulations (see table 1 in $\S 4.2$ ). In particular, cases with smaller numbers of pores are of interest to determine whether detrimental effects such as roughness/cavity-induced transition mechanisms are present.

The least-damped (or most unstable) eigenmode and the corresponding eigenvalue are determined by assuming (and verifying a posteriori) that the eigenvalues are well separated and fitting the long-time linear response to an exponentially damped or growing sinusoid.

\subsection{Linear stability analysis}

The LDNS results are compared to linear stability analysis, which is described by Fedorov (2010) and briefly reviewed here. We assume that the flow field $\boldsymbol{q}=[u, v, w, p, T]^{\mathrm{T}}$ can be decomposed into $\boldsymbol{q}=\overline{\boldsymbol{q}}+\boldsymbol{q}^{\prime}$, where $\overline{\boldsymbol{q}}$ is a base flow and the perturbation field $\boldsymbol{q}^{\prime}$ satisfies $\boldsymbol{q}^{\prime} \ll \overline{\boldsymbol{q}}$. Here, the velocity components $(u, v, w)$, the pressure $p$ and temperature $T$ are referenced to $U_{\infty}, \rho_{\infty} U_{\infty}^{2}$ and $T_{\infty}$, respectively. The Navier-Stokes equations are then linearized about $\overline{\boldsymbol{q}}$ by neglecting higher-order terms in $\boldsymbol{q}^{\prime}$ to give a first-order approximation. The parallel base flow is characterized by $\overline{\boldsymbol{q}}(y)$ taken from the DNS solution for the solid surface (i.e. steady disturbances induced by the cavities are neglected). The three-dimensional perturbation field $\boldsymbol{q}^{\prime}$ is expressed in the travelling form

$$
\boldsymbol{q}^{\prime}(x, y, z, t)=\operatorname{Re}(\hat{\boldsymbol{q}}(y) \exp [\mathrm{i}(\tilde{\alpha} x+\tilde{\beta} z-\Omega t)]),
$$

where $\tilde{\alpha}$ and $\tilde{\beta}$ are the wavenumbers, and $\Omega$ is the angular frequency. For spatial stability, $\Omega$ is taken to be a real and prescribed frequency $\omega_{S}$.

The system of stability equations for $\hat{\boldsymbol{q}}(y)$ is solved with the boundary conditions $(\hat{u}, \hat{v}, \hat{w}, \hat{p}, \hat{T}) \rightarrow 0$ for $y \rightarrow \infty$, and

$$
\hat{u}(0)=\hat{w}(0)=\hat{T}(0)=0, \quad \hat{v}(0)=A \hat{p}(0),
$$

where $A$ is the porous-layer admittance. On the solid wall (without cavities), the admittance is 0 , and $\hat{v}(0)=0$. On the porous wall, an approximate boundary condition is used, in which the admittance is expressed as

$$
A=\frac{\phi}{Z_{0}} \tanh (m H) .
$$

Recall that $\phi$ is the porosity and $H$ is the cavity depth. The terms $Z_{0}$ and $m$ are the characteristic impedance and propagation constant, which are expressed in terms of the 
complex dynamic density $\tilde{\rho}$ and complex compressibility $\tilde{C}$ as

$$
Z_{0}=\frac{1}{\rho_{\infty} U_{\infty}} \sqrt{\frac{\tilde{\rho}}{\tilde{C}}}, \quad m=\mathrm{i} \omega_{S} \sqrt{\tilde{\rho} \tilde{C}} .
$$

The parameters $\tilde{\rho}$ and $\tilde{C}$ are calculated using the solutions of Kozlov et al. (2005) for harmonic disturbances in a deep cavity. Their analytical expressions are

$$
\tilde{\rho}=\frac{\rho_{w} \Lambda}{\Lambda-\tan \Lambda}, \quad \tilde{C}=\frac{1}{\gamma p_{w}}\left[1+(\gamma-1) \frac{\tan \tilde{\Lambda}}{\tilde{\Lambda}}\right],
$$

where $\Lambda=\sqrt{\mathrm{i} \omega_{S} \rho_{w} b^{2} / \mu_{w}}$ characterizes the ratio of the cavity width to the Stokes layer thickness and $\tilde{\Lambda}=\sqrt{\operatorname{Pr}} \Lambda$. Recall that $b$ is the cavity half-width and that the subscript $w$ denotes a quantity at the wall $y=0$.

The stability equations are integrated from the outer boundary-layer edge to the wall using a fourth-order Runge-Kutta scheme and a Gram-Schmidt orthonormalization procedure. From known boundary conditions in the free stream, the eigenvalues are found using a shooting/Newton-Raphson procedure in order to satisfy the wall boundary conditions in (2.2). At convergence, the eigenvalues are related through the numerically obtained dispersion relation $D\left(\tilde{\alpha}, \tilde{\beta}, \omega_{S}\right)=0$.

In what follows, the linear stability analysis focuses on the second mode, which is the dominant instability in two-dimensional and quasi-two-dimensional boundary layers at hypersonic speed. Therefore, the parameter $\tilde{\beta}$ is set to 0 . The LST results for three-dimensional (oblique) waves confirm that the maximal instability is observed for $\tilde{\beta}=0$.

\section{Acoustic properties of porous coatings}

While viscous dissipation and heat conductivity inside individual cavities are accounted for in the LST model, end effects associated with scattering of incoming acoustic waves by the cavity mouth and small-scale disturbances generated near the UAC surface are not considered. Also, there is no coupling between disturbances in neighbouring cavities, which may not be true for closely spaced cavities.

In previous work by Brès et al. (2010), DNS were performed to clarify these effects and validate the robustness of the theoretical model by Kozlov et al. (2005) used in the approximate porous-wall boundary condition of the LST. For various acoustic Reynolds numbers $R e$ relevant to hypersonic flight, the reflection coefficient was computed as a function of the incident acoustic wave frequency and angle of incidence $\theta$, for coatings of different porosities $\phi$ and constant length-to-depth ratio $A R=0.12$, matching the aspect ratio of the cylindrical cavities used in the experiment by Rasheed et al. (2002). To further investigate the effect of the cavity depth and porosity, additional simulations and comparisons are performed in the present work. Typically, coatings with very deep pores $(A R<0.05)$ or very high porosity $(\phi>0.8)$ are not of interest for practical applications. Therefore, nine different coatings are considered here, for aspect ratio $A R=0.06,0.12,0.3$, and porosity $\phi=0.2,0.48,0.8$. These parameters span the range of typical coating designs used in most experimental and theoretical studies on UAC.

The reflection coefficient obtained from DNS is compared with the theoretical modelling in figure 2, for the nine different designs, at $R e=100$. For typical UAC parameters, the range of frequencies $0.1 \leqslant f H / a_{w} \leqslant 1.5$ contains the frequency of the 
most amplified second-mode waves. Much like in the previous study by Brès et al. (2010), the comparisons show good agreement between the two methods, especially for low aspect ratio and low frequency. Note that the theoretical model assumes that the cavity half-width $b$, depth $H$ and spacing $s$ satisfy the conditions $b \sim s \ll \lambda_{\text {acs }}$, and $H \sim \lambda_{a c s}$, where $\lambda_{\text {acs }}$ is the wavelength of an incident acoustic wave. Here, the discrepancies at $A R=0.3$ are probably due to the limitations of the theoretical assumptions.

Overall, the DNS results demonstrate that the theoretical model is an efficient and reliable method to provide estimates of the acoustic properties of the porous coatings. The analysis also shows that there is a critical acoustic Reynolds number $R e_{c r i t}$ for the presence of the cancellation/reinforcement regime, above which acoustic disturbances are not completely absorbed inside the pores. In that case, interference between incoming and outgoing (reflected from the cavity bottom) waves leads to a significant decrease of the reflection coefficient at some specific frequencies. As expected, $R e_{c r i t}$ is related to viscous and thermal absorption inside the pores and is only a function of the aspect ratio $A R$. From the theoretical modelling, the dependence of the critical Reynolds number on the aspect ratio is estimated as $R e_{\text {crit }} \approx 2 / A R$. As a result, for the aspect ratios considered in figure $2, R e=100>R e_{\text {crit }}$, and all the coatings exhibit local minima of the reflection coefficient. The results also confirm that the corresponding frequency of these local minima is largely independent of $A R, \phi$, and $R e$.

From figure 2, it is clear that the most efficient attenuative coating is obtained for $A R=0.06, \phi=0.8$ (i.e. curve with $\nabla$ symbols in figure $2 a$ ), which requires many deep pores. Such high porosity can be difficult to achieve in practical applications because of structural constraints. Also, the stabilizing effect may not be required for such a wide range of frequency. By restricting our attention to a smaller range of acoustic wave frequencies around the dominant second-mode frequency, similar or even better acoustic absorptive properties could be obtained with fewer and larger pores (e.g. curve with $\bigcirc$ symbols in figure $2(b)$ around $f H / a_{w}=0.7$ ). The stability of hypersonic boundary layers over UAC operating approximately in a local cancellation/reinforcement regime is investigated in the next section.

\section{Boundary-layer stability calculations}

\subsection{Spatial versus temporal stability}

To explore the validity of the temporal approach, the existing spatially evolving LST for a hypersonic boundary layer is reformulated to enable comparison with the present temporally evolving instability results.

The starting point is $(2.1)$ with $\tilde{\beta}=0$. For spatial stability, $\Omega$ is taken to be a real and prescribed frequency $\omega_{S}$, and the eigenvalue problem is solved for the complex eigenvalue $\tilde{\alpha}$. If $\operatorname{Im}(\tilde{\alpha})<0$, then the flow is unstable with spatial growth rate $\sigma_{S}=-\operatorname{Im}(\tilde{\alpha})$ and streamwise wavenumber $\alpha_{S}=\operatorname{Re}(\tilde{\alpha})$.

In our case, we are interested in the temporal stability: $\tilde{\alpha}$ is a real and prescribed streamwise wavenumber $\alpha_{T}$, and $\Omega$ is the complex eigenvalue. If $\operatorname{Im}(\Omega)>0$, then the flow is unstable with temporal growth rate $\sigma_{T}=\operatorname{Im}(\Omega)$ and frequency $\omega_{T}=\operatorname{Re}(\Omega)$. Eventually, the determination of the least-damped (or most-unstable) modes for a given wavelength $\alpha_{T}$ amounts to finding the eigenvalue $\Omega$ and corresponding eigenvector $\hat{\boldsymbol{q}}$ by integrating the governing equations directly in the time domain.

To a first approximation (valid for weakly unstable modes), the spatial and temporal formulation can be related using the Gaster (1962) transformation. In this 

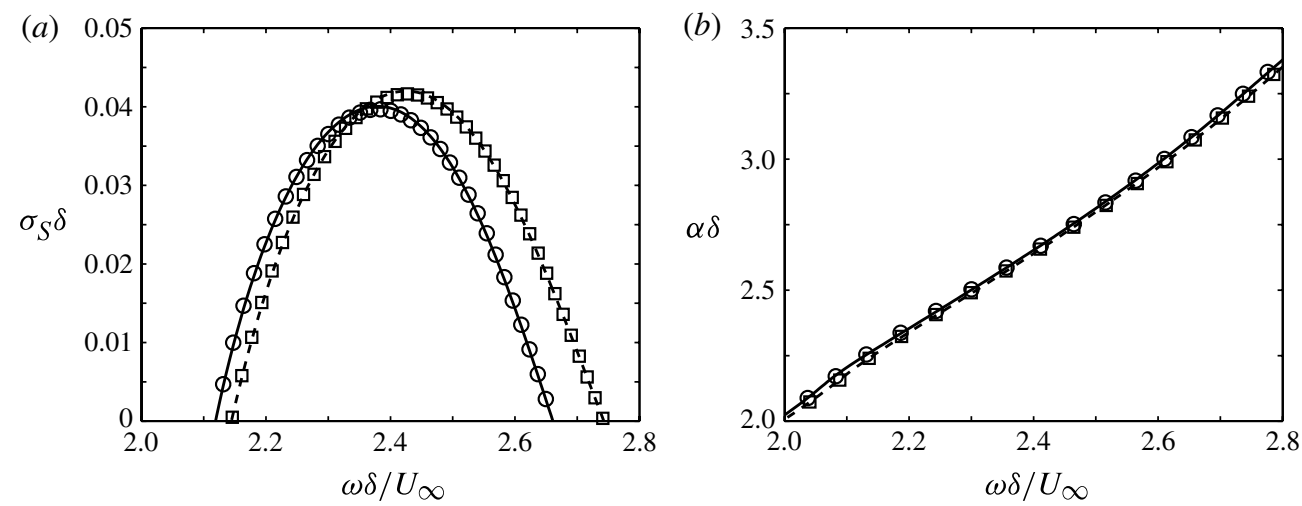

FIGURE 3. Comparison of the temporal and spatial linear stability analysis for the DNS boundary-layer profiles over a flat plate at $M_{\infty}=6, T_{w} / T_{\infty}=1.4$, for $R_{\delta}=24000(\bigcirc$, temporal; - $\longrightarrow 31300$ ( $\square$, temporalial) and $R_{\delta}=---$, spatial): (a) growth rate; (b) streamwise wavenumber. To enable comparison, the Gaster transformation has been applied to the temporal growth rate in $(a)$.

approximation, $\Omega$ is assumed to be an analytic function of $\tilde{\alpha}$, and the following equations are obtained by integration of the Cauchy-Riemann relations, and neglecting higher-order terms:

$$
\alpha_{T}=\alpha_{S}=\alpha, \quad \omega_{T}=\omega_{S}=\omega, \quad \sigma_{T}=\sigma_{S} C_{g}=\sigma,
$$

where $C_{g}=\partial \omega_{T} / \partial \alpha_{T}$ is the group velocity. The transformation requires that the (numerical) data for the eigenvalues be differentiated.

To validate the temporal growth approximation, a comparison is made between the results of LST for spatially and temporally growing instabilities. These calculations can be conducted for any locally parallel profile. Here, both spatial and temporal analyses are performed using the base flow compressible DNS solutions calculated for a flat plate at Mach $M_{\infty}=6$ and isothermal wall temperature $T_{w} / T_{\infty}=1.4$. The linear stability results are computed here for $\operatorname{Re}_{\delta}=\rho_{\infty} U_{\infty} \delta / \mu_{\infty}=24000$ and $\operatorname{Re}_{\delta}=31300$, where $\delta$ is the boundary-layer thickness defined as $U(y=\delta)=0.99 U_{\infty}$.

The comparisons, in non-dimensional form, between spatial and temporal growth rate, and between spatial and temporal streamwise wavenumber are shown in figures $3(a)$ and $3(b)$, respectively, as a function of frequency $\omega \delta / U_{\infty}$. To enable the comparison, the Gaster transformation has been applied to the temporal results.

Both spectra show good agreement over the range of unstable frequencies, demonstrating that the development of the second-mode instability proceeds in a similar fashion for both spatial and temporal boundary layers. Therefore, we conclude that the temporal formulation can be used to study UAC performance, and that results relevant to practical applications with spatially developing boundary layers can be obtained by applying the Gaster transformation to the temporal DNS results.

\subsection{The reinforcement regime}

We first consider the reinforcement regime by choosing parameters associated with the peaks in reflectance shown in figure 2. The parameters are summarized in table 1. 

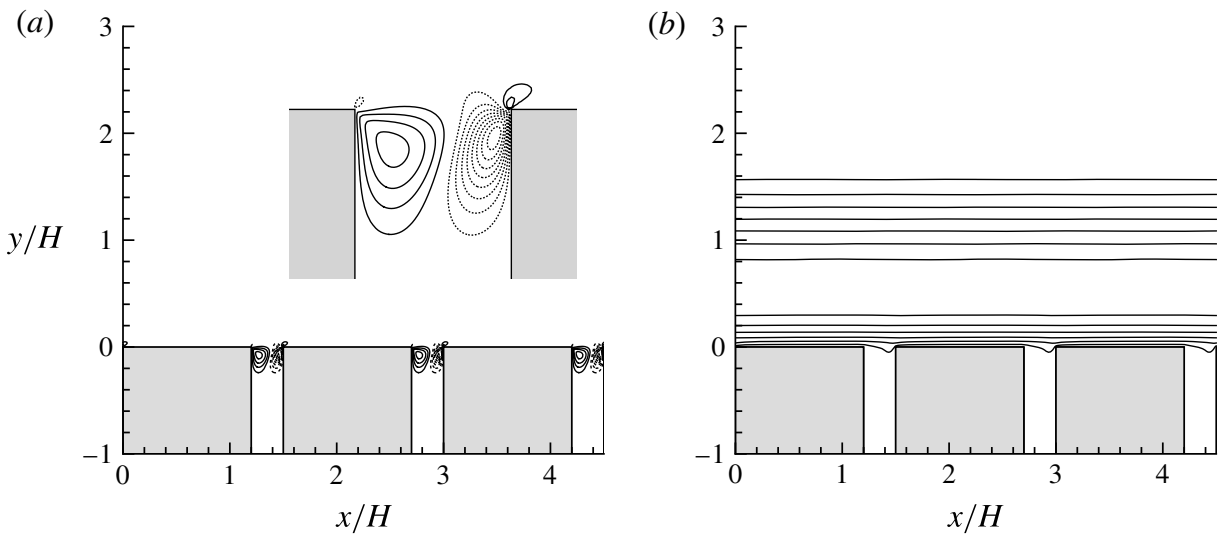

FIGURE 4. Base flow field from DNS for the hypersonic boundary layer at $M_{\infty}=6$ over a coating of porosity $\phi=0.2$, cavity aspect ratio $A R=0.3$ : (a) $v$, with zoom-in view near a pore; $(b) T$. Solid and dashed lines denote positive and negative velocity contours, respectively.

\begin{tabular}{|c|c|c|c|c|c|c|c|c|c|c|}
\hline & Flat plate & \multicolumn{3}{|c|}{$\mathrm{UAC} A R=0.06$} & \multicolumn{3}{|c|}{$\mathrm{UAC} A R=0.12$} & \multicolumn{3}{|c|}{$\mathrm{UAC} A R=0.3$} \\
\hline$T_{w} / T_{\infty}$ & 1.4 & & 1.4 & & & 1.4 & & & 1.4 & \\
\hline$M_{\infty}$ & 6 & & 6 & & & 6 & & & 6 & \\
\hline$\delta / H$ & - & & 2 & & & 2 & & & 2 & \\
\hline$R e_{\delta}$ & 24000 & & 24000 & & & 24000 & & & 24000 & \\
\hline$R e$ & - & & 36 & & & 72 & & & 181 & \\
\hline$R e_{\text {crit }}$ & - & & 33 & & & 17 & & & 7 & \\
\hline$\phi$ & - & 0.2 & 0.48 & 0.8 & 0.2 & 0.48 & 0.8 & 0.2 & 0.48 & 0.8 \\
\hline$L_{x} / \delta$ & 2.5 & 2.4 & 2.5 & 2.475 & 2.4 & 2.5 & 2.475 & 2.25 & 2.5 & 2.4375 \\
\hline$N_{\text {pore }}$ & - & 16 & 40 & 66 & 8 & 20 & 33 & 3 & 8 & 13 \\
\hline
\end{tabular}

TABLE 1. Parameters for the DNS of a temporally evolving boundary layer over a flat plate and over nine different coatings of varying porosity $\phi$ and cavity aspect ratio $A R$ in the reinforcement regime ( - denotes not applicable).

All the linear stability calculations in this section are performed at Reynolds number $R e_{\delta}=24000$, which is related to the acoustic Reynolds number $R e=\rho_{w} a_{w} b / \mu_{w}$ by

$$
\operatorname{Re}=\operatorname{Re}_{\delta} \frac{A R}{2 M_{\infty}} \frac{H}{\delta}\left(\frac{T_{\infty}}{T_{w}}\right)^{n+1 / 2},
$$

with $n=1$ to take into account the temperature dependence of viscosity modelled in the current study.

Contours of normal velocity and temperature are shown in figure 4 for a coating of porosity $\phi=0.2$, cavity aspect ratio $A R=0.3$. Recall that these results are from nonlinear DNS, and are used as base flow in the linear simulations of this coating. While the boundary-layer profile over the flat plate is a function of only the normal direction, the flow field with UAC is two-dimensional. The negative and positive normal velocities at the downstream and upstream cavity edges, respectively, indicate 

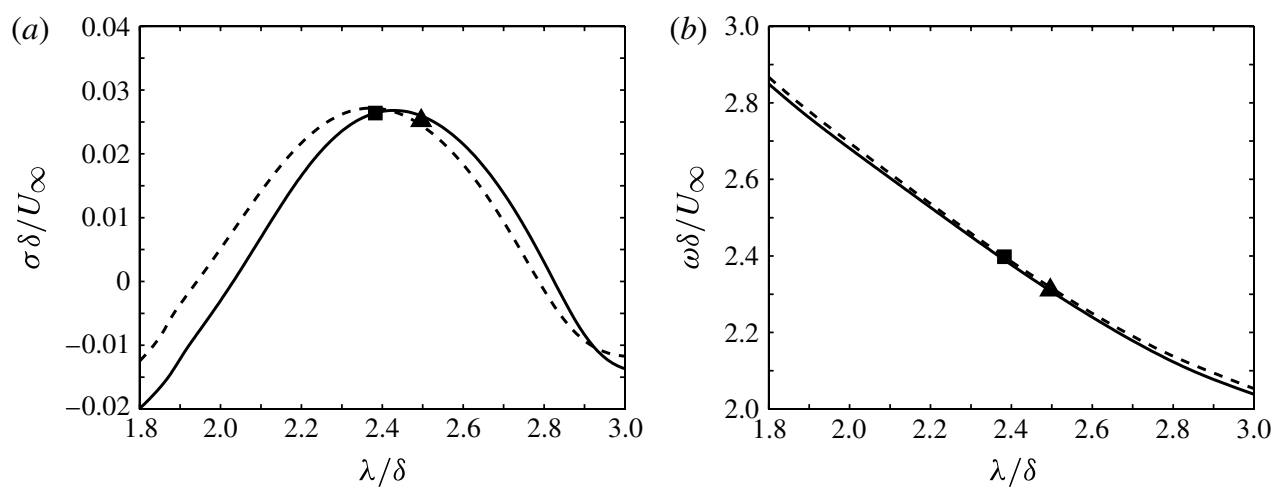

FIGURE 5. Second-mode instability for a hypersonic boundary layer over a flat plate at $M_{\infty}=6, R_{\delta}=24000$ (— LST; $\_$, LDNS), and $R e_{\delta}=31300$ (----, LST; $\mathbf{\square}$, LDNS): (a) growth rate; (b) frequency.

weak recirculation within the pores. In contrast, the temperature is essentially uniform inside the pores, equal to the wall temperature. Because the pores are small compared to the boundary-layer thickness, the recirculating flow near the top of the cavity is essentially steady, and there is no Rossiter-type feedback mechanism nor global instabilities observed in the simulations.

The LST predictions for the frequency response of the boundary layer are obtained by applying the stability calculations from Fedorov et al. (2001) to twodimensional second-mode waves at $R e_{\delta}=24000$. These calculations are conducted for the boundary-layer profile extracted from the corresponding DNS of the flow over a flat plate. For the UAC configurations, the approximate porous-wall boundary condition discussed in $\S 2.2$ is used to account for the presence of the pores. The growth/decay rate and frequency of the least-damped (or most-unstable) mode are computed as a function of the streamwise wavelength $\lambda / \delta$.

The LST results for the hypersonic boundary layer over a flat plate (i.e. no cavities) at $R e_{\delta}=24000$ are presented in figure 5. The second-mode has a positive growth rate for streamwise wavelengths $2.03 \leqslant \lambda / \delta \leqslant 2.81$, with the most unstable mode at $\lambda / \delta=2.42$. This motivated the choice of $L_{x} / \delta \approx 2.5$ as the streamwise extent of the computational domain. The predicted linear growth rate for the second-mode instability at $\lambda / \delta=2.5$ is $\sigma \delta / U_{\infty}=2.52 \times 10^{-2}$, and the LDNS results match the linear calculation. The typical second-mode structures are shown in figures $6(a)$ and $6(b)$ for normal velocity $v^{\prime}$ and temperature $T^{\prime}$, respectively. Here, the prime denotes the deviation from the frozen base flow. Similarly to the results in Sandham \& Lüdeke (2009), the maximum perturbation is near the wall for the temperature, and near $y=0.35$ for the normal velocity. This maximum of the velocity perturbation falls near the critical layer of the base flow at $y=0.3$, as expected from linear theory (Mack 1969, 1984).

The acoustic reflectance properties of the different UAC considered here are plotted in figure 7. The range of unstable frequencies (shaded grey) and the most unstable second mode (vertical solid line) for the hypersonic boundary layer over the flat plate at $R e_{\delta}=24000$ are also shown in the figure. Here, the frequency of the dominant second mode is $\omega \delta / U_{\infty}=2.36$, which can be expressed as a function of the acoustic 
(a)

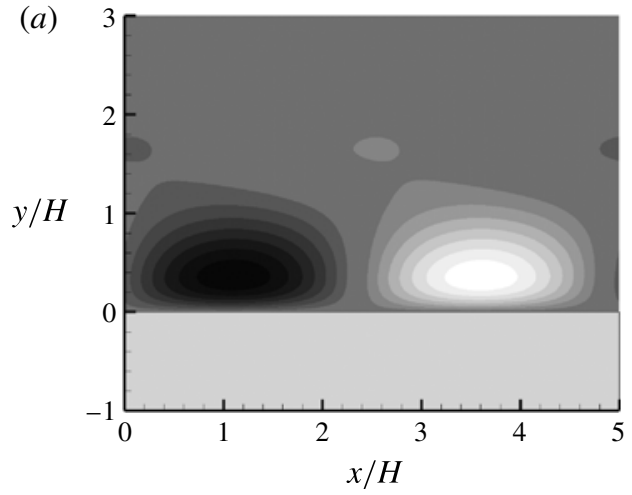

(b)

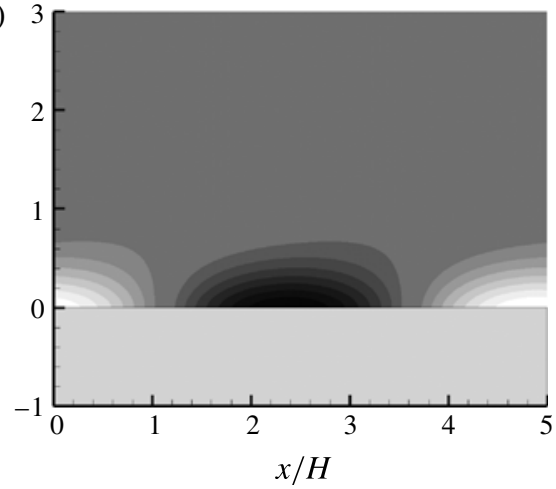

FIGURE 6. Perturbation field for a hypersonic boundary layer over a flat plate at $M_{\infty}=6$, $R e_{\delta}=24000$ (from LDNS), showing the structures of the second-mode instability: $(a) v^{\prime}$; (b) $T^{\prime}$.
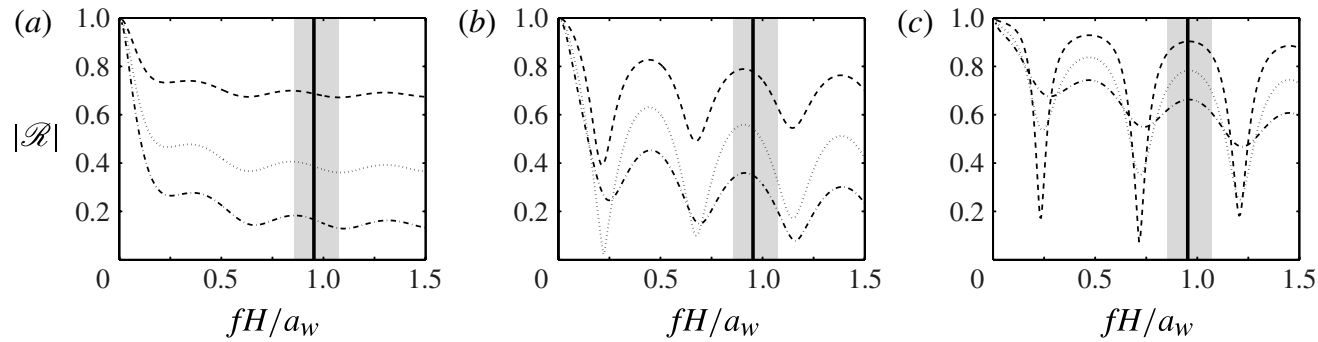

FIGURE 7. Reflection coefficient amplitude at normal incidence $(\theta=0)$ from theory, for the coatings of porosity $\phi=0.2(----), \phi=0.48(\cdots \cdots)$, and $\phi=0.8(-\cdot-)$ in the reinforcement regime in table 1: $(a)$ cavity aspect ratio $A R=0.06 ;(b) A R=0.12 ;(c) A R=0.3$. The grey shaded area corresponds to the frequencies of the unstable modes from figure $5(b)$, and the vertical thick line is the frequency of the most unstable mode.

frequency $f H / a_{w}$ using

$$
\frac{f H}{a_{w}}=\frac{1}{2 \pi} \frac{\omega \delta}{U_{\infty}} M_{\infty} \sqrt{\frac{T_{\infty}}{T_{w}}} \frac{H}{\delta} .
$$

As shown in figure 7 , the second-mode frequency corresponds approximately to the acoustic frequency of a maximum of the reflection coefficient for all the configurations. These conditions were chosen to quantify the different UAC performance in the reinforcement regime (i.e. the 'worst-case scenario'). Since the operating conditions of a uniform UAC are expected to depend on the streamwise position and alternate between local minima and maxima of the coating acoustic absorption, it is important to make sure that the stabilizing effect is obtained even for unfavourable conditions.

The LST predictions for the growth rate are compared to the LDNS results in figures $8(a), 8(b)$ and $8(c)$ for the UAC of aspect ratio $A R=0.06, A R=0.12$ and $A R=0.3$, respectively. A similar comparison is presented for the frequencies in figure 9. There is again good agreement between the two methods, with less than $5 \%$ error on the growth rate and less than $1 \%$ difference in the frequencies for all 

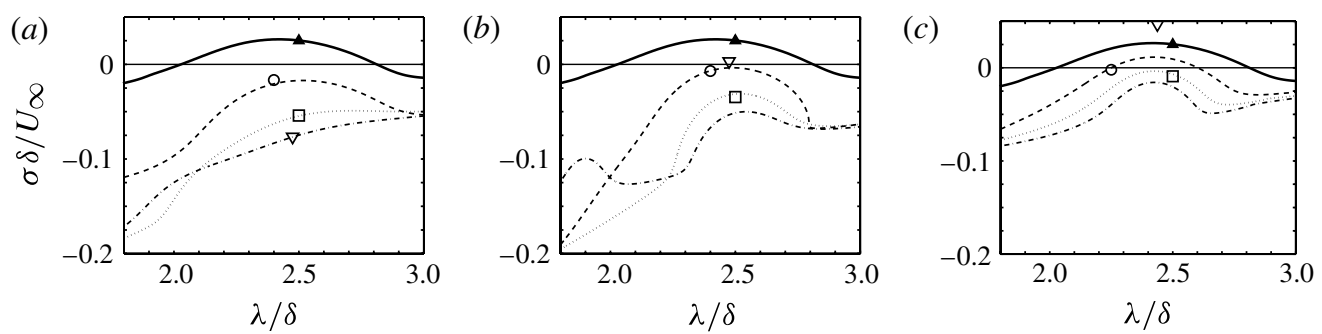

FIGURE 8. Linear growth rate $\sigma$ of the most unstable mode for a hypersonic boundary layer at $M_{\infty}=6$ and $R e_{\delta}=24000$ over a flat plate (-, LST; $\boldsymbol{\Delta}$, LDNS) and over coatings of porosity $\phi=0.2(----$, LST; $\bigcirc$, LDNS), $\phi=0.48(\cdots$, , LST; $\square$, LDNS), and $\phi=0.8$ $(-\cdot-$ LST; $\nabla$, LDNS): (a) cavity aspect ratio $A R=0.06 ;(b) A R=0.12 ;(c) A R=0.3$.
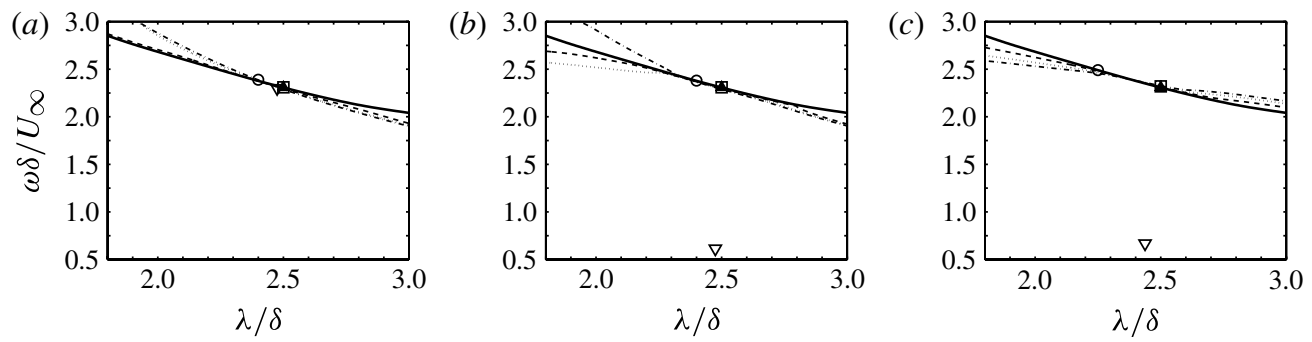

FIGURE 9. Frequency $\omega$ of the most unstable mode for a hypersonic boundary layer at $M_{\infty}=6$ and $R e_{\delta}=24000$ over a flat plate (—, LST; $\mathbf{\Delta}$, LDNS) and over coatings of porosity $\phi=0.2(----$, LST; $\bigcirc$, LDNS), $\phi=0.48(\cdots)$, LST; $\square$, LDNS), and $\phi=0.8$ $(-\cdot-$ LST; $\nabla$, LDNS): (a) cavity aspect ratio $A R=0.06 ;(b) A R=0.12 ;(c) A R=0.3$.

cases, with the notable exception of $\phi=0.8(\nabla)$, which is further discussed below. In contrast to the growth rate, the presence of the porous coatings of different porosity and cavity aspect ratio has little effect on the frequency. As previously discussed, the second-mode frequency is related to the boundary-layer thickness, which is the same for the different simulations.

Even in this configuration corresponding to a minimum of the coating acoustic absorption, almost all the UAC geometries successfully stabilize the second mode, demonstrating the robustness of such coatings. The exception is the case of a coating of large pores $(A R=0.3)$ and low porosity $(\phi=0.2)$ which still exhibits a small positive growth rate in figure $8(c)$, for both the LST (----) and LDNS (O). As expected, the very low number of pores per wavelength of instability $\left(N_{\text {pore }}=3\right)$ and the poor acoustic scattering performance $(|\mathscr{R}|>0.9$ at the second-mode frequency in figure $7 c$ ) of this particular design lead to a less efficient coating. The velocity and temperature perturbation fields for the $A R=0.3, \phi=0.2$ geometry are shown in figure 10. The second-mode structures for the boundary layer over this geometry are still visible, reminiscent of the results for the flat plate in figure 6. Besides the largescale structure relevant to the second mode, the perturbation field also exhibits smallerscale structures associated with the interaction of the boundary-layer disturbance with the cavities, and with acoustic waves emanating from the cavity openings.

Finally, in the case of high porosity $\phi=0.8(\nabla)$, the higher grow rate and very low frequency suggest a different instability altogether. Here, the LDNS results highlight 

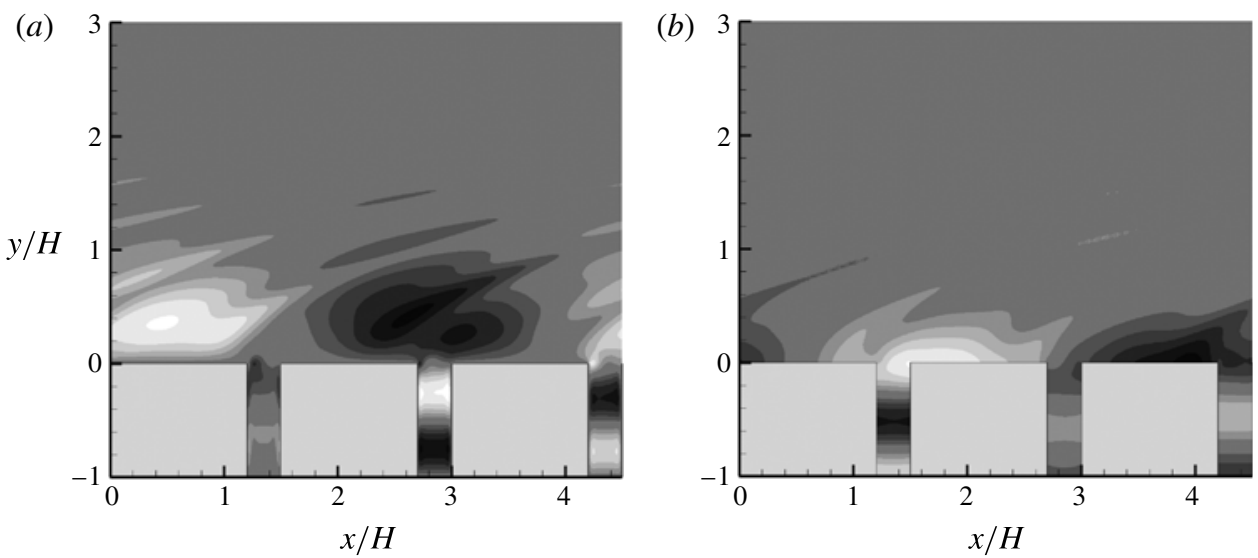

FIGURE 10. Perturbation field for a hypersonic boundary layer over a coating of porosity $\phi=0.2$ and cavity aspect ratio $A R=0.3$, at $M_{\infty}=6, R e_{\delta}=24000$ (from LDNS): (a) $v^{\prime}$; (b) $T^{\prime}$.

Flat plate $\quad \mathrm{UAC} A R=0.12$

$\begin{array}{lcccc}T_{w} / T_{\infty} & 1.4 & & 1.4 & \\ M_{\infty} & 6 & & 6 & \\ \delta / H & - & & 2.6 & \\ \operatorname{Re}_{\delta} & 31300 & & 31300 & \\ \operatorname{Re} & - & & 94 & \\ \operatorname{Re}_{\text {crit }} & - & & 17 & \\ \phi & - & 0.2 & 0.48 & 0.8 \\ L_{x} / \delta & 2.6 & 2.54 & 2.60 & 2.60 \\ N_{\text {pore }} & - & 11 & 27 & 45\end{array}$

TABLE 2. Parameters for the DNS of a temporally evolving boundary layer over a flat plate and over three different coatings of porosity $\phi$ and cavity aspect ratio $A R$ in the cancellation regime (- denotes not applicable).

the presence of an acoustic slow mode which destabilizes the flow and causes it to differ substantially from the theoretical second-mode value. This leads to outright instability for the UAC geometry $A R=0.3, \phi=0.8$ in figure $8(c)$. This new mode is discussed in $\S 5$.

\subsection{The cancellation regime}

To model the optimum cancellation regime, the nonlinear simulations are continued until the boundary-layer thickness reaches $\delta / H=2.6$, so that the linear stability calculations can be performed at Reynolds number $R e_{\delta}=31300$. The parameters are summarized in table 2 . The LST results for the hypersonic boundary layer over the flat plate are compared to those for $\operatorname{Re}_{\delta}=24000$ in figure 5. The change in wavelength of the most unstable mode between the two cases is not significant. This is because the reference length scale for the data is chosen to be the boundary-layer thickness $\delta$ and for the second mode of maximal growth, $\lambda / \delta$ is almost constant. Likewise, because the second-mode frequency can be well predicted using the inviscid theory, the changes in frequency are minimal. In contrast, the growth rate is affected by viscosity, and the 

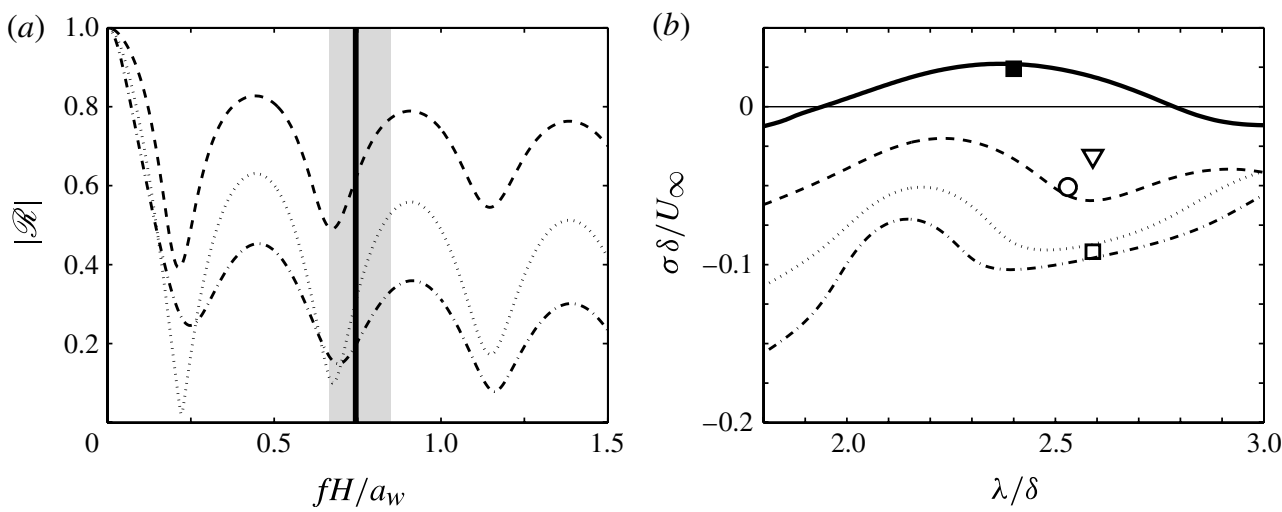

FIGURE 11. Performance improvement for the coatings in the cancellation regime in table 2: $M_{\infty}=6, R e_{\delta}=31,300$, cavity aspect ratio $A R=0.12$ and porosity $\phi=0.2(----; \bigcirc)$, $\phi=0.48(\cdots \cdots ; \square)$ and $\phi=0.8(-\cdot-; \nabla) .(a)$ Reflection coefficient amplitude from theory. The grey shaded area corresponds to the frequencies of the unstable modes from figure $5(b)$, and the vertical thick line is the frequency of the most unstable mode. (b) Linear growth rate from LST (lines) and LDNS (symbols), for the UAC and the flat plate (—; $)$ ).

maximum value increased slightly. For $R e_{\delta}=31300$, the second mode has a positive growth rate for wavelengths $1.94 \leqslant \lambda / \delta \leqslant 2.78$. The most-unstable mode has frequency $\omega \delta / U_{\infty}=2.40$ and occurs at $\lambda / \delta=2.37$.

Unlike the stability results, the acoustic Reynolds number and the acoustic scattering properties of the coating are independent of the boundary-layer thickness and unchanged. Using (4.3), the frequency of the most unstable second mode now approximately corresponds to a local minimum of the reflection coefficient at normal incidence $(\theta=0)$, as shown in figure 11(a). These conditions correspond to a cancellation regime (i.e. best-case scenario), where the UAC operates at a local maximum of acoustic absorption. The corresponding linear growth rates are presented in figure 11(b). As anticipated, a significant stabilizing effect is obtained for this configuration, over a large range of wavelength, compared to the results in figure $8(b)$. Note that in the case of high porosity $\phi=0.8$, the new acoustic slow mode is still present.

\subsection{Nonlinear simulations}

Nonlinear numerical simulations including the outer flow are performed for the UAC configurations in the reinforcement regime, to confirm the linear results and investigate potential detrimental effects of the coating. Following the same procedure as for the linear simulations, the same pressure-pulse perturbation is added to the flow and the long-time response is recorded. For each of the cases considered in table 1, the nonlinear simulations agreed fully with the results of linear analysis. That is, except for the particular case of $\phi=0.8$ and $A R=0.3$ discussed in the next section, the nonlinear simulations all resulted in damped second-mode response and the boundary layer remained laminar. For the coating of aspect ratio $A R=0.3$ and porosity $\phi=0.8$, where a new unstable mode is observed in the linear response of the perturbed boundary layer, the same instability growth is present in the nonlinear simulation, with similar frequency and growth rate as the LDNS results. Here, the nonlinear simulations confirm that this configuration exhibits a dominant instability of growth rate larger than that of the most unstable second mode. The current DNS would need 


$\begin{array}{ccccccccc}\phi & A R & R e_{\delta} & \alpha \delta & \sigma \delta / U_{\infty} & \omega \delta / U_{\infty} & C_{i} / U_{\infty} & C_{r} / U_{\infty} & f H / a_{w} \\ 0.8 & 0.12 & 24000 & 2.54 & 3.15 \times 10^{-3} & 0.614 & 1.24 \times 10^{-3} & 0.242 & 0.248 \\ 0.8 & 0.3 & 24000 & 2.58 & 4.65 \times 10^{-2} & 0.669 & 1.80 \times 10^{-2} & 0.259 & 0.270\end{array}$

TABLE 3. Simulation parameters and characteristics of the new unstable mode $R$ predicted by LDNS for coatings of porosity $\phi=0.8$ and cavity aspect ratio $A R=0.12$ and 0.3 . The corresponding acoustic frequency $f H / a_{w}$ is computed using (4.3).

to be extended to longer times to investigate whether the mode eventually reaches an asymptotic state, limit cycle or continues to evolve into more complicated fluctuations.

It should be mentioned that the present study of the nonlinear effects of the porous coating has several limiting assumptions. First, only strictly two-dimensional, planar cavities are considered. Second, the periodic domain was fixed to approximately one wavelength of the dominant second mode. Thus, any first-mode instability would not be captured, even if they were unstable, since their wavelength is longer than permitted in the computational domain. With these limitations in mind, the nonlinear DNS results did not show differences with the linearized simulations, and there was no evidence of strong interactions between the cavity flow and the second mode (aside from the stabilizing effects already discussed), nor roughness/cavity-induced transition mechanisms (even for small numbers of pores per wavelength of instability, as low as 3 ). Here, it is important to recall that the pores are relatively deep and narrow, and small compared to the boundary-layer thickness: the flow physics inside the cavities is dominated by viscosity, and feedback mechanisms or global instabilities are not present.

\section{Analysis of the high-porosity unstable mode}

As noted above, numerical simulations of high-porosity UAC geometries $(\phi=0.8)$ revealed an unexpected unstable mode. For this instability, the growth rate $\sigma \delta / U_{\infty}$, the frequency $\omega \delta / U_{\infty}$ and complex phase speed $C / U_{\infty}$ obtained from LDNS are presented in table 3, where $C=C_{r}+\mathrm{i} C_{i}=(\omega+\mathrm{i} \sigma) / \alpha$, and $\alpha=2 \pi / \lambda$ is the wavenumber. As discussed in $\S 4.2$, the frequency is much smaller than that of a Mack second mode. A comparison of the structure of this instability obtained from LDNS in figure 12 to that of the second mode (e.g. figure 10) also indicates that the instability wave structures are distinct.

\subsection{Theory}

To clarify the properties of the new resonant mode, referred hereafter as mode $R$, we compare LST results of the disturbance spectrum for the solid-wall case to the porouswall case of porosity $\phi=0.8$ and cavity aspect ratio $A R=0.3$. For the solid-wall case in figure 13, the spectra $\omega(\alpha)$ and $\sigma(\alpha)$ are typical for a hypersonic boundary layer (e.g. Fedorov \& Khokhlov 2001; Fedorov \& Tumin 2011). At small wavenumbers the fast mode $F$ (solid thick line) coalesces with fast acoustic waves of phase speed $c=1+1 / M_{\infty}$, while the slow mode $S$ (dashed line) coalesces with slow acoustic waves of $c=1-1 / M_{\infty}$. Owing to the synchronization of these modes in the vicinity of $\alpha \delta \approx 2.4$, the branching of the dispersion relation leads to destabilization of mode $F$ and stabilization of mode $S$. Here the unstable mode $F$ corresponds to the Mack second mode. 

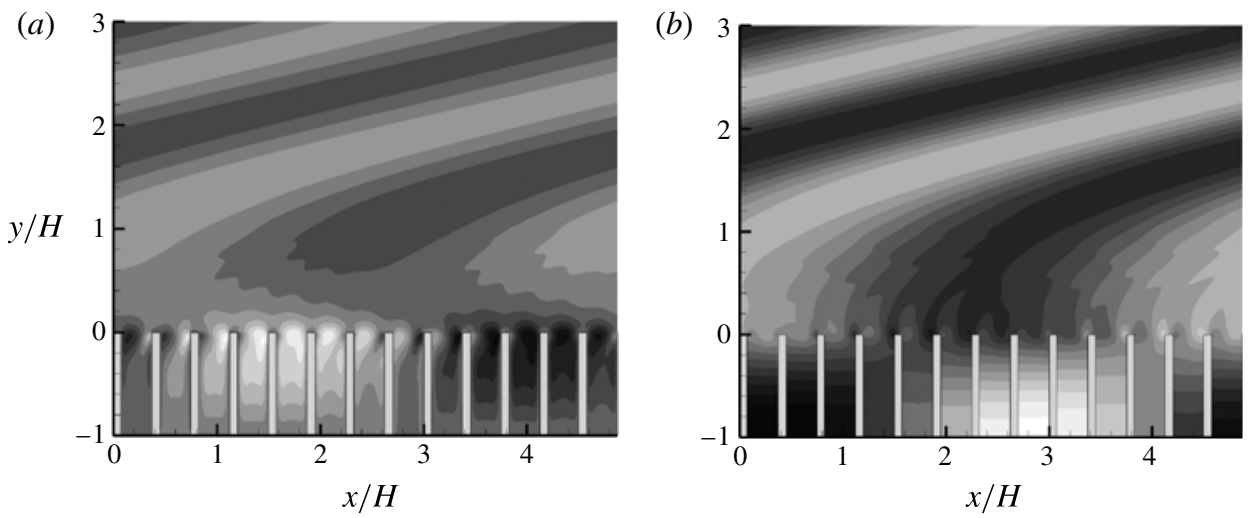

FIGURE 12. Structure of the new mode instability $R$ for the hypersonic boundary layer over a coating of porosity $\phi=0.8$ and cavity aspect ratio $A R=0.3$, at $M_{\infty}=6, R e_{\delta}=24000$ (from LDNS): $(a) v^{\prime}$; (b) $P^{\prime}$.
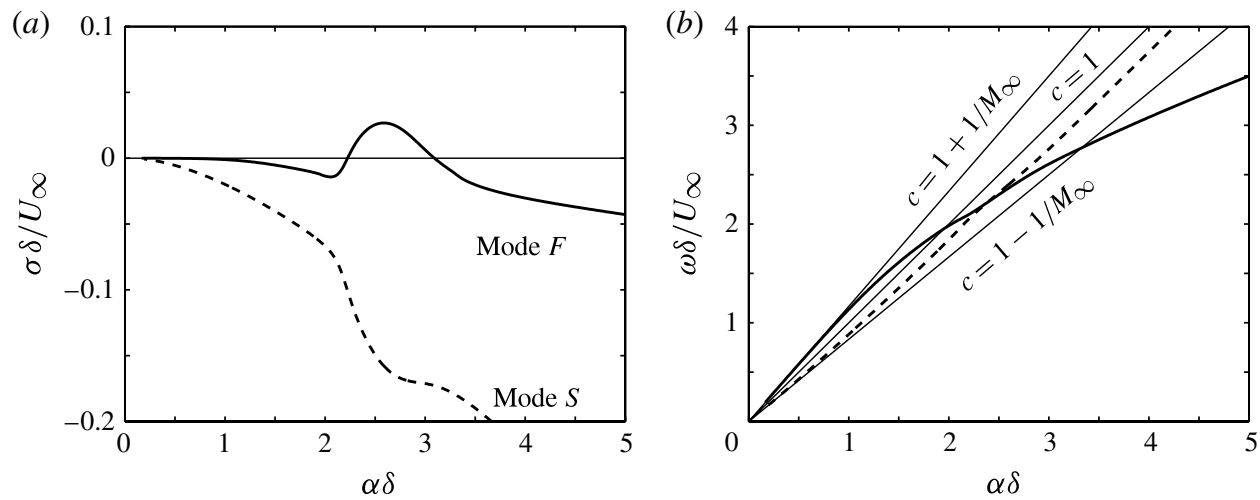

FIGURE 13. Disturbance spectrum $\sigma(\alpha)$ and $\omega(\alpha)$ for the solid wall at $\operatorname{Re}_{\delta}=24000$ : modes $F(-)$ and $S(----)$.

For the porous-wall case of $\phi=0.8$ and $A R=0.3$ in figure 14 , the situation changes dramatically. Besides the previous dispersion lines, which relate to modes $F$ and $S$ on the solid wall for $\alpha \delta>2$, there are new modes, in particular mode $R$ shown by the thick black line. As in the solid-wall case, branching of the dispersion relation near the synchronization points leads to destabilization of one mode and stabilization of the other. Namely, the synchronization between modes $F_{1}$ and $S_{1}$ leads to the instability of mode $S_{1}$ at relatively small $\alpha$. This instability is not observed in the solid-wall case. Furthermore, the new mode $R$ is unstable in a wide wavenumber range and has growth rate larger than that of mode $F$ in the solid-wall case. The spectral line $\omega(\alpha)$ of mode $F_{1}$ (line with circles) is overlapped by the spectral line of mode $R$ (thick black line) in figure 14(b), while their growth rates remain essentially different in figure 14(a). This behaviour of the spectrum becomes clearer when the spectral lines are plotted in the complex-phase-speed plane shown in figure 15. As $\alpha$ increases, the phase speed of mode $F_{1}$ departs from the fast acoustic branch, crosses the vorticity/entropy branch and approaches the slow acoustic branch. As soon as mode $F_{1}$ coalesces with the lower side of the slow acoustic branch, mode $R$ departs from the upper side of this branch. 

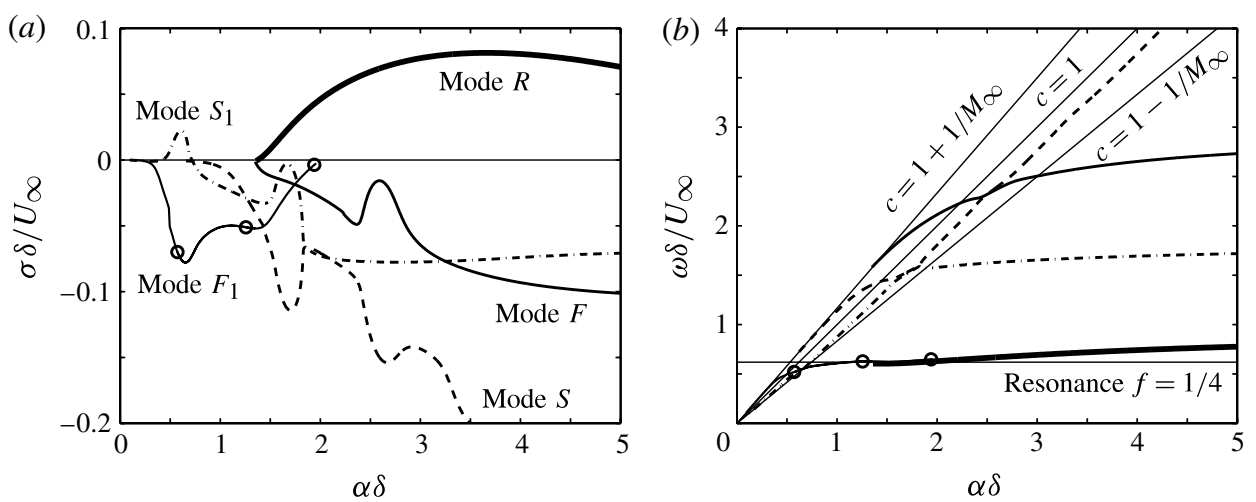

FIGURE 14. Disturbance spectrum $\sigma(\alpha)$ and $\omega(\alpha)$ for the porous wall of porosity $\phi=0.8$ and cavity aspect ratio $A R=0.3$ at $R e_{\delta}=24000:$ modes $F(-), S(----), F_{1}(-\bigcirc-), S_{1}$ $(-\cdot-)$ and new mode $R(-)$.

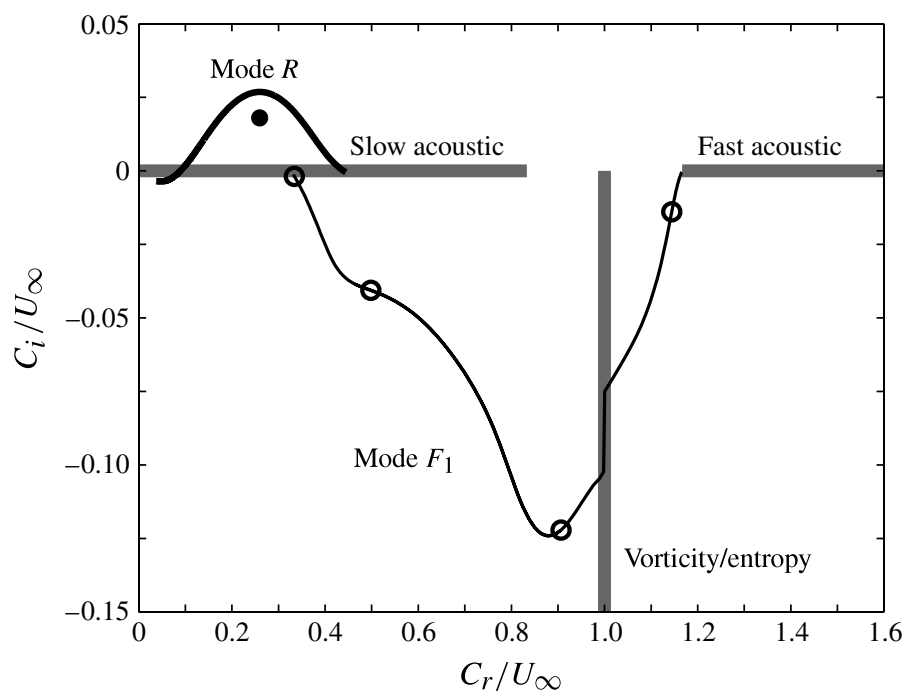

FIGURE 15. LST Spectral lines of mode $F_{1}\left(-\bigcirc_{-}\right)$and mode $R(-)$ and LDNS results (-) for the porous wall of porosity $\phi=0.8$ and cavity aspect ratio $A R=0.3$ at $R e_{\delta}=24000$, in the complex-phase-speed plane $\left(C_{r}, C_{i}\right)$. The thick grey lines show the branches of slow acoustic waves $\left(C_{r} / U_{\infty}<1-1 / M_{\infty}, C_{i} / U_{\infty}=0\right)$, fast acoustic waves $\left(C_{r} / U_{\infty}>1+1 / M_{\infty}, C_{i} / U_{\infty}=0\right)$ and vorticity/entropy waves $\left(C_{r} / U_{\infty}=1, C_{i} / U_{\infty} \leqslant 0\right)$ in the inviscid approximation.

The pressure perturbation field in figure $12(b)$ indicates that in each cavity the pressure antinode is at the cavity bottom and the pressure node is at the cavity top, consistent with resonant forcing within the cavity. The first resonance of an isolated, deep cavity $(b \ll H)$ occurs at the acoustic frequency $f H / a_{w}=1 / 4$. In this case, each cavity works as an acoustic resonator that is excited at the lowest frequency corresponding to a wavelength of $4 H$. The acoustic frequency $f$ corresponding to the frequency $\omega$ of the new mode $R$ can be computed using (4.3). As shown in table 3, this frequency is close to the first acoustic resonance. 

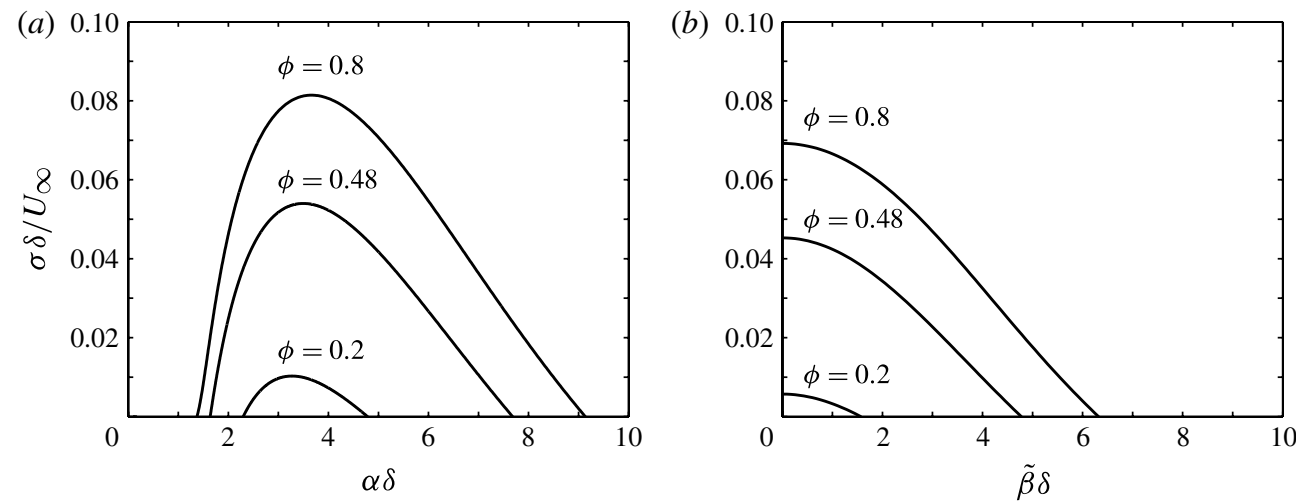

FIGURE 16. The growth rate of mode $R$ for coatings of different porosity $\phi$ at $\operatorname{Re}_{\delta}=24000$ and cavity aspect ratio $A R=0.3:(a)$ at lateral wavenumber $\tilde{\beta}=0 ;(b)$ at streamwise wavenumber $\alpha \delta=2.58$.

Therefore, it is natural to assume that the new instability is associated with resonant interactions in the cavities. To shed light on the physics of such a system of coupled resonating cavities, we consider the simple case of a two-dimensional inviscid wave propagating over a porous surface without external flow. For such a system, the phase speed can be shown (see the Appendix for details) to be

$$
\frac{C_{r}}{a_{w}}=\frac{1}{\sqrt{1+\phi^{2} \tan ^{2}\left(2 \pi f H / a_{w}\right)}} .
$$

Thus, an increase in the porosity of the coating leads to a decrease of the disturbance phase speed. As $f$ approaches the resonance condition, the phase speed tends to zero. Thus, the disturbance fields near the cavity mouths are coupled such that the near-surface wave can propagate at much slower speeds than acoustic waves in an unbounded medium. Under such resonance conditions, the UAC impedance is small, and the porous structure may support a surface wave with small phase speed, consistent with the observed new mode $R$. For long-wave disturbances with frequency significantly smaller than the first-resonance frequency, cavities do not resonate, and the wave is stable.

As shown in figure $16(a)$, mode $R$ is stabilized as porosity decreases. Presumably this is due to the fact that the vertical velocity amplitude averaged over the porous surface decreases proportionally to the porosity $\phi$. The LST results for threedimensional (oblique) waves related to the mode $R$ are shown in figure $16(b)$ for $\alpha \delta=2.58$ corresponding to the conditions in table 3. As expected for acoustic instability of a hypersonic boundary layer, the maximal growth rate is observed for two-dimensional waves having the lateral wavenumber $\tilde{\beta}=0$.

Figure 17 illustrates the cavity depth effect, where the frequency $\omega$ and the growth rate $\sigma$ of mode $R$ are shown as functions of the cavity aspect ratio $A R$. These calculations were carried out at the wavenumber $\alpha \delta=2.58$, porosity $\phi=0.8$ and the acoustic Reynolds number $R e=181$ related to the conditions in table 3. Deep cavities $(A R<0.125)$ and shallow cavities $(A R>0.475)$ do not resonate, and mode $R$ is stable. The maximum instability is observed for cavities of moderate aspect ratio $A R \approx 0.3$ favourable for the resonance mechanism. The results show that the unstable region 


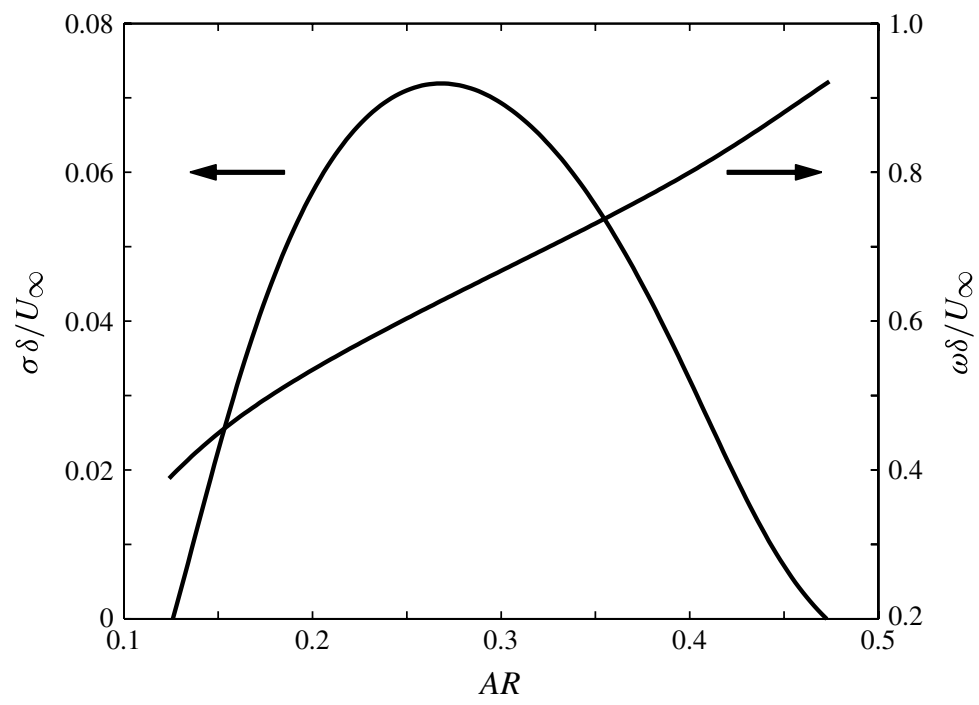

FIGURE 17. The distributions $\sigma(A R)$ and $\omega(A R)$ for mode $R$ at porosity $\phi=0.8$, streamwise wavenumber $\alpha \delta=2.58$, and acoustic Reynolds number $R e=181$.
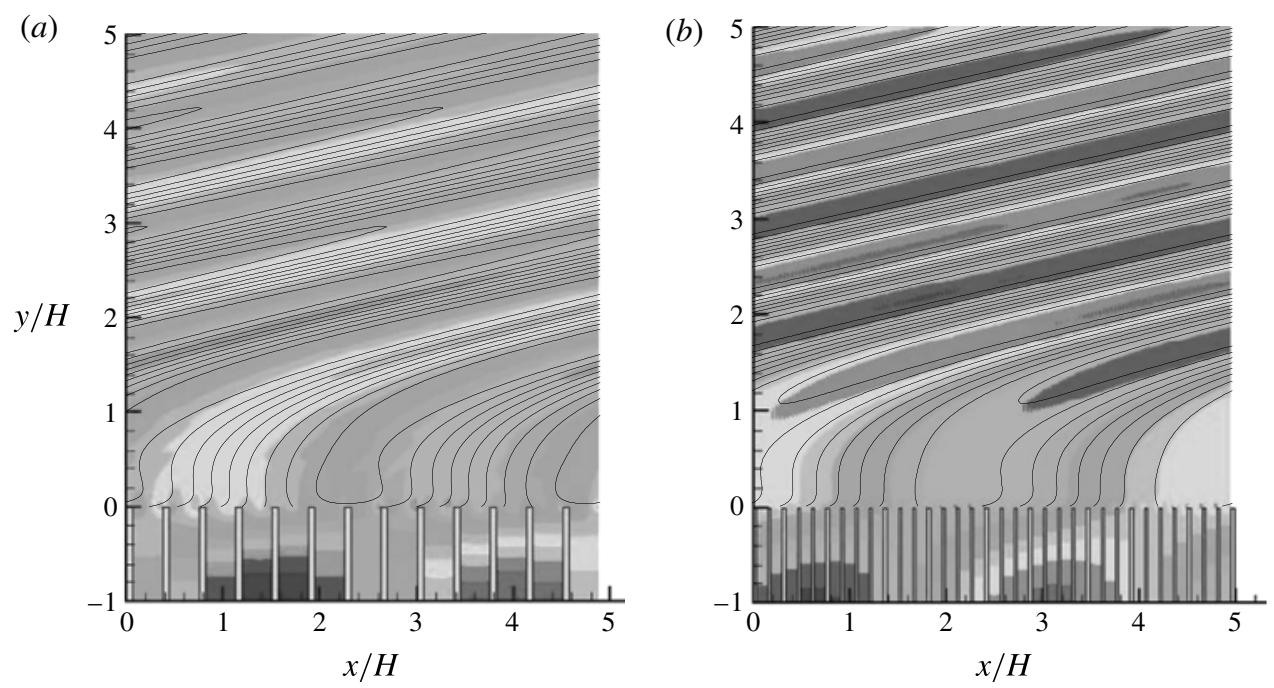

FIGURE 18. Comparison of pressure perturbation field from LST (black lines) and LDNS (grey contours) for coatings of porosity $\phi=0.8$ at $R e_{\delta}=24000$ : (a) cavity aspect ratio $A R=0.3$ and streamwise wavenumber $\alpha \delta=2.58 ;(b) A R=0.12$ and $\alpha \delta=2.54$.

is formed in the vicinity of the resonance point $H_{\text {res }}$, with the maximum growth rate located at $H / H_{\text {res }} \approx 1.1$.

\subsection{Comparison to LDNS}

As shown in figure 18, the instantaneous pressure disturbance field predicted by LST (black lines) closely matches the structure of the disturbance field obtained through LDNS (flooded contours) for the case $\phi=0.8$, with cavity aspect ratios $A R=0.3$ and $A R=0.12$, respectively. Figure 19 shows that the LDNS frequency values closely 

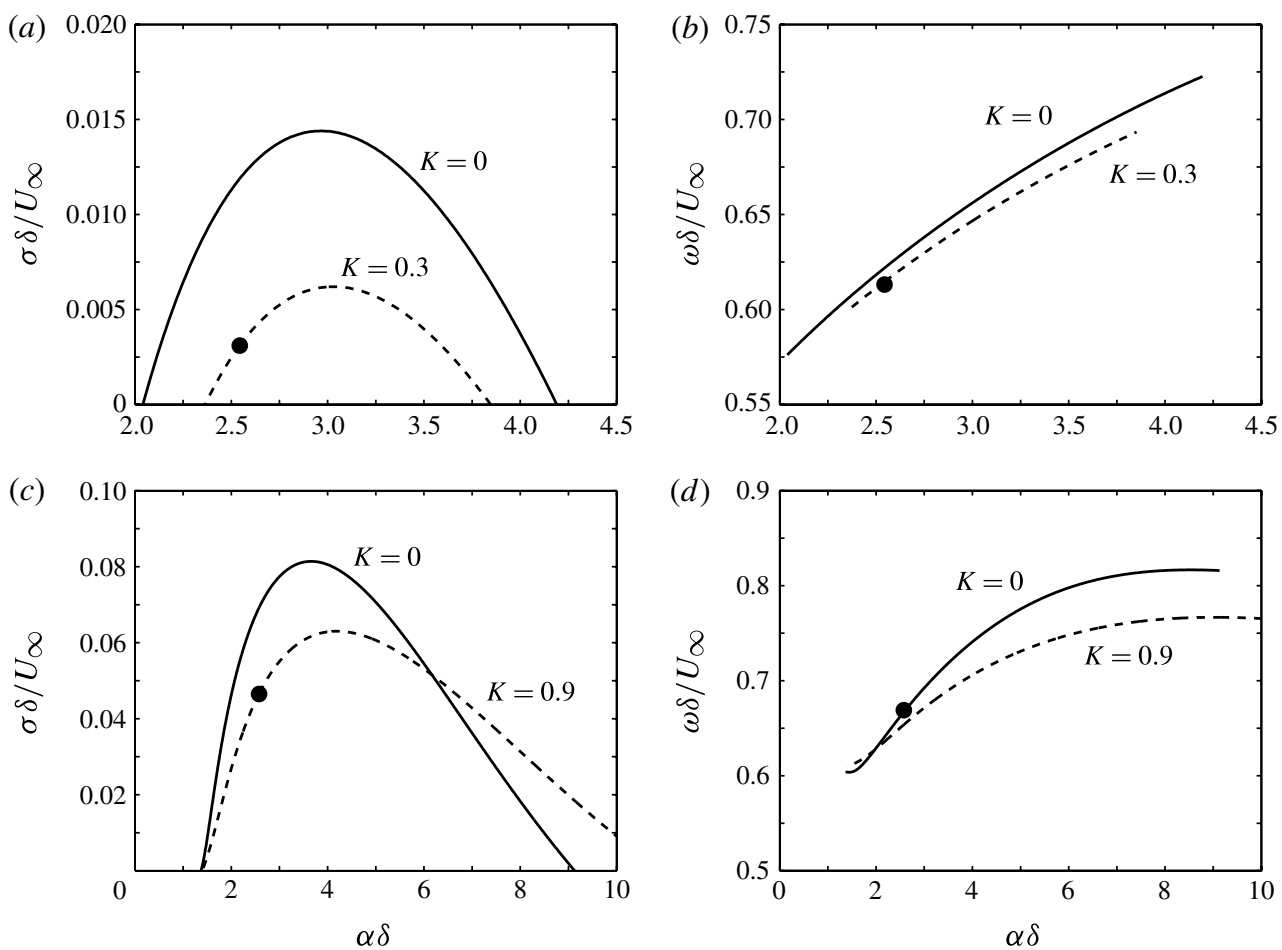

FIGURE 19. Growth rate $\sigma(\alpha)$ and frequency $\omega(\alpha)$ of mode $R$ for coatings of porosity $\phi=0.8$, cavity aspect ratio $A R=0.12(a, b)$, and $A R=0.3(c, d)$. No-slip boundary condition $u(0)=0$ (solid lines); boundary condition in (5.5) (dashed line); LDNS results ( $)$.

match the theoretically predicted values, but the growth rates are substantially lower (compare the curves marked $K=0$ with the symbols). It appears that some effect not accounted for in the theory is retarding the growth of this mode.

We propose that the discrepancy is due a slip velocity associated with the influence of the cavity mouth on the coupling between disturbances in neighbouring cavities, as the presence of such flow structures is clear in figure $4(a)$. The presence of a non-zero streamwise velocity disturbance $u \neq 0$ at $y=0$ may affect the growth rate of the new mode $R$. To check this hypothesis, we consider the linearized equation for the $x$-momentum with an inviscid approximation

$$
\rho\left(\frac{\partial u}{\partial t}+U \frac{\partial u}{\partial x}+v \frac{\partial U}{\partial y}\right)=-\frac{\partial p}{\partial x} .
$$

In the derivation of (5.2) we utilize the fact that in the mean flow, $U=U(y)$ and $V=0$. Substituting in a disturbance of the form $q=\hat{q}(y) \exp (\mathrm{i} \alpha x-\mathrm{i} \omega t)$ and setting $\rho=1 / T$, we obtain

$$
\frac{1}{T}\left(-\mathrm{i} \omega \hat{u}+\mathrm{i} \alpha U \hat{u}+\hat{v} U^{\prime}\right)=-\mathrm{i} \alpha \hat{p}
$$

where prime denotes the derivative $\mathrm{d} / \mathrm{d} y$. 
On those portions of $y=0$ outside the cavities, $\hat{u}(0)=0$, while along the cavity mouths the momentum equation gives free slip,

$$
\hat{u}(0)=\frac{-\mathrm{i} U^{\prime}(0) \hat{v}(0)+\alpha T(0) \hat{p}(0)}{\omega} .
$$

In order to account for this in the LST, we modify the boundary condition by merging these two expressions for $\hat{u}(0)$ with the porosity, obtaining

$$
\hat{u}(0)=\frac{K \phi}{\omega}\left(-\mathrm{i} U^{\prime}(0) \hat{v}(0)+\alpha T(0) \hat{p}(0)\right),
$$

where $K$ is a tunable parameter that we use to account for viscous effects that will further reduce the slip velocity, but which are difficult to model from first principles owing to the complex near-cavity flow field. As the reduction in velocity $\hat{u}(0)$ should increase as the cavity width decreases, we expect $K$ to decrease with $A R$.

Parametric calculations made using this model show that, for the coating of porosity $\phi=0.8$ and cavity aspect ratio $A R=0.12$, LST results with $K=0.3$ agree well with the DNS results (figure $19 a, b$ ). For the case $\phi=0.8, A R=0.3$, the cavity is wider by a factor of 2.5 , and the theory with $K=0.9$ agrees well with DNS (figure $19 c, d$ ). Thus the variation of the $K$ parameter follows the expected behaviour, increasing as the cavity aspect ratio increases. It should be noted that (5.5) and the accompanying calculations are offered as an explanatory analysis rather than a predictive one; further refinements would be required to predict the appropriate value of $K$ as a function of $A R$.

\section{Conclusions}

In this work, the stabilizing effect of ultrasonic absorptive coatings on hypersonic boundary-layer instabilities has been investigated with linear and nonlinear solutions of the two-dimensional Navier-Stokes equations, and linear stability theory. Our previous work on acoustic scattering properties of two-dimensional porous coatings and previous LST results motivate a consideration of UAC designs which have not been systematically studied in the past.

The coatings considered consist of a uniform array of two-dimensional rectangular pores (slots) of aspect ratios $A R=0.06,0.12,0.3$, and porosity $\phi=0.2,0.48$, and 0.8. The conservative way to design coatings is to have the UAC operated in an attenuative regime where the pores are relatively deep and acoustic waves (and second-mode instabilities) are attenuated by viscous effects inside the pores. Here, alternative designs are also considered, where the pores are relatively shallow and the coating operates in the alternating cancellation/reinforcement regime, which is related to reflections of acoustic waves from the bottom of the cavities, and dependent on the frequency of the acoustic waves.

For the numerical simulations of the boundary layer at Mach number $M_{\infty}=6$, a computationally tractable and efficient temporal method is used to account for the detailed flow physics associated with micro-cavities that attenuate second-mode instabilities in hypersonic boundary layers. Here, temporally (rather than spatially) evolving boundary layers are considered. This greatly reduces computational expense, since it allows the computational domain to include a single wavelength of the instability (which still may require $\sim 20$ micro-cavities in the simulation). We provide evidence that this approximation is appropriate for slowly growing second-mode 
instabilities and that spatial instability results can be accurately recovered from the temporal ones via the Gaster transformation.

With the exception of one case discussed below, the linear DNS confirm the results of LST that employs an approximate porous-wall boundary condition. Both methods show that the UAC stabilizing performances are directly related to their acoustic scattering performances. At Reynolds numbers $R e_{\delta}=24000$, most of these UAC configurations correspond to the reinforcement regime where the second-mode frequency matches a frequency of minimum absorptive properties of the coating (i.e. 'worst-case scenario'). It is important to consider these cases to ensure that the UAC stabilizing effect is obtained even in unfavourable conditions. To model the optimum cancellation regime where the UAC operates at a local maximum of acoustic absorption (i.e. 'best-case scenario'), the LDNS and LST are performed at Reynolds numbers $\operatorname{Re}_{\delta}=31300$. As expected, a significant stabilizing effect is obtained for these configurations over a large range of wavelength.

While coatings currently used in applications are typically uniform, and in the attenuative regime, this analysis suggests that the theoretical acoustic modelling could be used to optimize the pore depth as a function of the streamwise direction. The dependence arises from the fact that the second-mode properties depend on the streamwise location, that is, at each $x$-station, there are several unstable waves of slightly different frequencies, as pointed out by Fedorov et al. (2001). By enforcing an optimum streamwise-dependent depth, the coating could potentially always operate at the maximum absorption of the frequency of the local most-unstable second mode.

As mentioned, the LDNS uncovered a new unstable mode on the wall with coatings of sufficiently high porosity. This mode propagates downstream with low phase speed and has a growth rate which can be larger than that of the most unstable second mode. The new instability was also captured by the LST with the impedance boundary conditions on the porous surface. The baseline theoretical model, which assumes zero fluctuations of the longitudinal velocity on the porous surface, predicts the basic trends of the new instability and helps to explain its physical mechanism. Namely, this theory shows that the new resonant mode, referred to as mode $R$, is associated with acoustic resonances in the cavities. Disturbance fields near the mouths of resonating cavities are coupled such that the boundary-layer disturbance is decelerated and becomes unstable. Parametric studies indicate that the new instability occurs on coatings having closely spaced cavities of moderate aspect ratio. To avoid this detrimental effect, which can reduce the UAC laminar-flow-control performance, the coating should have sufficiently small porosity and/or narrow pores of sufficiently small aspect ratio. Restrictions on these parameters can be estimated using the baseline theoretical model. However, these estimates may be too conservative, because the theory overpredicts the mode $R$ growth rate. It was shown that this overshoot is associated with non-zero fluctuations of the longitudinal velocity on the porous surface. To get more accurate restrictions on the UAC parameters, one should perform direct numerical simulations or develop a theoretical model accounting for this slip effect.

Note that previous theoretical, numerical and experimental studies of the UAC stabilization effect have been focused on the low-porosity and/or narrow-pore configurations, which do not provide favourable conditions for the foregoing instability mechanism. Moreover, actual porous coatings have low porosities because of the structural constraints and chances to face the new instability are small. Nevertheless, optimal UAC (providing maximal damping of the Mack second mode) have high porosity and relatively shallow cavities. Seeking this optimum, one should check that the coating does not sustain the new instability. 
Finally, nonlinear simulations of the same cases were performed and led to the same conclusions as the linear analysis. The porous coatings damp second-mode instability and the boundary layer remains laminar. Additionally, the nonlinear results did not exhibit any 'tripping' of the boundary layer by small-scale disturbances associated with individual pores.

\section{Acknowledgements}

This work was supported by the Air Force of Scientific Research, and partially (A.V. and F.) by the AFOSR/NASA National Center for Hypersonic Research in Laminar-Turbulent Transition. Computer time was provided in part by the Department of Defense High Performance Computing centres.

\section{Appendix.}

Consider a two-dimensional inviscid wave propagating over a porous surface without external flow, with the $+y$ direction corresponding to the outgoing normal to the surface. Over the wall, the pressure disturbance $p=\hat{p}(x, y) \exp (-\mathrm{i} \omega t)$ is governed by the Helmholtz equation

$$
\Delta \hat{p}+\left(\frac{\omega}{a_{w}}\right)^{2} \hat{p}=0,
$$

which has the general solution

$$
\begin{gathered}
\hat{p}(x, y)=\hat{p}^{+} \exp (\mathrm{i} \alpha x+\kappa y)+\hat{p}^{-} \exp (\mathrm{i} \alpha x-\kappa y), \\
\kappa^{2}=\alpha^{2}-\frac{\omega^{2}}{a_{w}^{2}}=\alpha^{2}\left(1-\frac{C^{2}}{a_{w}^{2}}\right), \quad \operatorname{Re}(\kappa)>0 .
\end{gathered}
$$

For a wave propagating with the phase speed $C_{r}<a_{w}$, we have

$$
\kappa=\alpha \sqrt{1-\frac{C_{r}^{2}}{a_{w}^{2}}}
$$

for $\alpha>0$. Since the near-wall wave decays as $y \rightarrow \infty$, we obtain

$$
\hat{p}(x, y)=\hat{p}^{-} \exp (\mathrm{i} \alpha x-\kappa y) .
$$

Substituting the solution into the linearized $y$-momentum equation

$$
\frac{\partial v}{\partial t}+\frac{1}{\rho_{w}} \frac{\partial p}{\partial y}=0
$$

leads to

$$
-\mathrm{i} \omega \hat{v}^{-}-\frac{\kappa}{\rho_{w}} \hat{p}^{-}=0,
$$

which can be written as

$$
\omega=\frac{\mathrm{i} \kappa}{\rho_{w}} Z(\omega),
$$

where $Z(\omega)=\hat{p}^{-} / \hat{v}^{-}$is the porous-wall impedance. Using the boundary condition at the cavity bottom $v(y=-H)=0$ and neglecting viscous effects inside the cavities, 
$Z$ is given by

$$
Z(\omega)=-\mathrm{i} \frac{\rho_{w} a_{w}}{\phi} \cot \left(\omega H / a_{w}\right) .
$$

Substituting the expression for $Z$ in (A 9) and the definition for $\kappa$ in (A4) into the dispersion relation in (A 8), the wave phase speed is given by

$$
\frac{C_{r}}{a_{w}}=\frac{1}{\sqrt{1+\phi^{2} \tan ^{2}\left(\omega H / a_{w}\right)}} .
$$

\section{REFERENCES}

Bountin, D. A., Shiplyuk, A. N., Maslov, A. A. \& ChokAni, N. 2004 Nonlinear aspects of hypersonic boundary-layer stability on a porous surface. AIAA Paper 2004-0255.

BRÈs, G. A. \& COLONIUS, T. 2008 Three-dimensional instabilities in compressible flow over open cavities. J. Fluid Mech. 599, 309-339.

Brès, G. A., Colonius, T. \& Fedorov, A. V. 2008 a Interaction of acoustic disturbances with micro-cavities for ultrasonic absorptive coatings. AIAA Paper 2008-3903.

Brès, G. A., Colonius, T. \& Fedorov, A. V. $2008 b$ Stability of temporally evolving supersonic boundary layers over micro-cavities for ultrasonic absorptive coatings. AIAA Paper 2008-4337.

Brès, G. A., Colonius, T. \& Fedorov, A. V. 2010 Acoustic properties of porous coatings for hypersonic boundary-layer control. AIAA J. 48 (2), 267-274.

Brès, G. A., Inkman, M., Colonius, T. \& Fedorov, A. V. 2009 Alternate designs of ultrasonic absorptive coatings for hypersonic boundary layer control. AIAA Paper 2009-4217.

Colonius, T., Lele, S. K. \& Moin, P. 1993 Boundary conditions for direct computation of aerodynamic sound. AIAA J. 31 (9), 1574-1582.

De Tullio, N. \& Sandham, N. D. 2010 Direct numerical simulation of breakdown to turbulence in a Mach 6 boundary layer over a porous surface. Phys. Fluids 22, 094105.

Fedorov, A. V. 2010 Temporal stability of hypersonic boundary layer on porous wall: comparison of theory with DNS. AIAA Paper 2010-1242.

Fedorov, A. V. 2011 Transition and stability of high-speed boundary layers. Annu. Rev. Fluid Mech. 43, 79-95.

Fedorov, A. V. \& KhoKhlov, A. P. 2001 Prehistory of instability in hypersonic boundary layer. Theor. Comput. Fluid Dyn. 14, 359-375.

Fedorov, A. V., Kozlov, V. F., Shiplyuk, A. N., Maslov, A. A., Sidorenko, A. A., Burov, E. V. \& MALmuth, N. D. 2003a Stability of hypersonic boundary layer on porous wall with regular microstructure. AIAA Paper 2003-4147.

Fedorov, A. V., Malmuth, N. D., Rasheed, A. \& Hornung, H. G. 2001 Stabilization of hypersonic boundary layers by porous coatings. AIAA J. 39 (4), 605-610.

Fedorov, A. V., Shiplyuk, A. N., Maslov, A. A., Burov, E. V. \& Malmuth, N. D. $2003 b$ Stabilization of a hypersonic boundary layer using an ultrasonically absorptive coating. J. Fluid Mech. 479, 99-124.

Fedorov, A. V. \& Tumin, A. 2011 High-speed boundary-layer instability: old terminology and a new framework. AIAA J. 49 (8), 1647-1657.

FREUND, J. B. 1997 Proposed inflow/outflow boundary condition for direct computation of aerodynamic sound. AIAA J. 35 (4), 740-742.

Gaster, M. 1962 A note on the relation between temporally-increasing and spatially-increasing disturbances in hydrodynamic stability. J. Fluid Mech. 14, 222-224.

HADER, C. \& FASEL, H. F. 2011 Numerical investigation of porous walls for a Mach 6.0 boundary layer using an immersed boundary method. AIAA Paper 2011-3081.

KENDALL, J. M. 1975 Wind tunnel experiments relating to supersonic and hypersonic boundary layer transition. AIAA J. 13 (3), 290-299.

Kimmel, R. 2003 Aspects of hypersonic boundary layer transition control. AIAA Paper 2003-0772. 
Kozlov, V. F., Fedorov, A. V. \& Malmuth, N. D. 2005 Acoustic properties of rarefied gases insides pores of simple geometries. J. Acoust. Soc. Am. 117 (6), 3402-3412.

LELE, S. K. 1992 Compact finite difference scheme with spectral-like resolution. J. Comput. Phys. 103, 16-42.

Mack, L. M. 1969 Boundary-layer stability theory. In Part B. Doc. 900-277. Jet Propulsion Lab, CA.

MACK, L. M. 1984 Boundary layer linear stability theory. In AGARD-R-709 Special Course on Stability and Transition of Laminar Flow, pp. 3.1-3.81.

Malmuth, N. D., Fedorov, A. V., Shalaev, V., Cole, J., Khokhlov, A., Hites, M. \& WILLIAMS, D. 1998 Problems in high speed flow prediction relevant to control. AIAA Paper 98-2695.

Maslov, A. A., Shiplyuk, A. N., Sidorenko, A. A., Polivanov, P., Fedorov, A. V., Kozlov, V. F. \& MALMUTH, N. D. 2006 Hypersonic laminar flow control using a porous coating of random microstructure. AIAA Paper 2006-1112.

Poinsot, T. J. \& LELE, S. K. 1992 Boundary conditions for direct simulations of compressible viscous flows. J. Comput. Phys. 101, 104-129.

Rasheed, A., Hornung, H. G., Fedorov, A. V. \& Malmuth, N. D. 2002 Experiments on passive hypervelocity boundary layer control using an ultrasonically absorptive surface. AIAA J. 40 (3), 481-489.

SAndham, N. D. \& LÜDEKE, H. 2009 A numerical study of Mach 6 boundary layer stabilization by means of a porous surface. AIAA J. 47 (9), 2243-2252.

SChlichting, H. \& Gersten, K. 2000 Boundary Layer Theory, 8th edn. Springer.

Stetson, K. F. \& Kimmel, R. G. 1992 Example of second-mode instability dominance at a Mach number of 5.2. AIAA J. 30 (12), 2974-2976.

Stetson, K. F., Thomson, E. R., Donaldson, J. C. \& Siler, L. G. 1983 Laminar boundary-layer stability experiments on a cone at Mach 8, Part 1: sharp cone. AIAA Paper 83-1761.

Thompson, K. W. 1990 Time-dependent boundary conditions for hyperbolic systems, II. J. Comput. Phys. 89, 439-461.

WARtemann, V., LÜDEKe, H. \& SAndham, N. D. 2009 Stability analysis of hypersonic boundary layer flow over microporous surfaces. AIAA Paper 2009-7202. 